\title{
Poisson kernel and Green function of balls for complex hyperbolic Brownian motion
}

\author{
by \\ TOMASZ ŻAK (Wrocław)
}

\begin{abstract}
The aim of this paper is to give a description of the Poisson kernel and the Green function of balls in the complex hyperbolic space. The description is in terms of the hypergeometric function and unitary spherical harmonics in $\mathbb{C}^{n}$.
\end{abstract}

1. Introduction. The Poisson kernel and Green function are the main objects in potential theory. Even in the classical context, i.e. for the Laplace operator $\Delta$ in $\mathbb{R}^{n}$, explicit formulas for the Poisson kernel $P_{D}(x, y)$ and the Green function $G_{D}(x, y)$ are known only for a few classes of sets $D$, including balls and half-spaces. Our main goal is to describe the Poisson kernel and the Green function of balls $B_{r}, 0<r<1$, in the complex hyperbolic space, that is, in the unit ball in $\mathbb{C}^{n}$ equipped with the Bergman metric and the Laplace-Beltrami operator $\Delta_{\mathrm{LB}}$. In our computations we use stochastic analysis, hence we first recall the probabilistic interpretation of the Poisson kernel and the Green function.

By the general theory, the Laplace-Beltrami operator $\Delta_{\mathrm{LB}}$ in the unit ball of $\mathbb{C}^{n}$ is the generator of a diffusion $\left(X_{t}\right)_{t \geq 0}$ with trajectories in this ball. We call this process the complex hyperbolic Brownian motion. For $r \in(0,1)$ let $B_{r}=\left\{z \in \mathbb{C}^{n}:|z|<r\right\}$ and let $\tau_{r}=\inf \left\{t>0:\left|X_{t}\right|=r\right\}$ be the first time the process $\left(X_{t}\right)_{t \geq 0}$, starting from $x \in B_{r}$, exits from $B_{r}$. Then the distribution of $X_{\tau_{r}}$ is the Poisson kernel for the ball $B_{r}$. We denote it by $P_{r}(x, y)$. Next consider $\left(X_{t}^{B_{r}}\right)_{t \geq 0}$, the process starting from $x \in B_{r}$ and killed on exiting $B_{r}$. Let $p_{B_{r}}(t, x, y)$ be the density of its transition probability. Then $G_{r}(x, y)=\int_{0}^{\infty} p_{B_{r}}(t, x, y) d t$ is the Green function of the ball $B_{r}$. Observe that $\Delta_{\mathrm{LB}}$ commutes with the action of the unitary group $U(n)$ ([R, Thm. 4.1.2]). This implies that if $\left(X_{t}\right)_{t \geq 0}$ starts from $x \in B_{r}$ then both

2000 Mathematics Subject Classification: Primary 60J45, 31C12, 60G15; Secondary $60 \mathrm{~J} 65$. motion.

Key words and phrases: Poisson kernel, Green function, complex hyperbolic Brownian

Research partially supported by KBN grant 1 P03A 02028. 
$P_{r}(x, y)$ and $G_{r}(x, y)$, as functions of $y$, are invariant with respect to those $g \in U(n)$ that leave $x$ fixed. This property allows us to use unitary spherical harmonics to examine $P_{r}(x, y)$ and $G_{r}(x, y)$. We will provide formulas for $P_{r}(x, y)$ and $G_{r}(x, y)$ in terms of the hypergeometric function ${ }_{2} F_{1}$ and complex unitary spherical harmonics.

In the case of real hyperbolic Brownian motion such formulas have recently been found by Byczkowski and Małecki $[\mathrm{BM}]$. For the complex hyperbolic space the Poisson kernel of the whole space (known as the Poisson-Szegö kernel for the unit ball) is well-known $([\mathrm{Kr}],[\mathrm{R}],[\mathrm{S}])$. In 1975 Folland $[\mathrm{F}]$ gave a description of this kernel in terms of spherical harmonics. The problem of finding the Green function is much harder-knowing the Green function we can easily compute the Poisson kernel by differentiation in the normal direction (cf. Theorem 8, p. 174 in [Ch] and Lemma 11 in the Appendix). The potential of the process $\left(X_{t}\right)_{t \geq 0}$ was computed in 2001 by Matsumoto [M]. Matsumoto computed the $\lambda$-potential, that is, $G^{\lambda}(x, y)=\int_{0}^{\infty} e^{-\lambda t} p_{t}(x, y) d t$ for all $\lambda \geq 0$, but, as he wrote (p. 554 of [M]), "in order to use stochastic analysis, we adopt the upper half-space realizations of the hyperbolic spaces". In this paper we prefer the ball realization of the complex hyperbolic space because in this case the hyperbolic Brownian motion is invariant with respect to the group $U(n)$ of unitary transformations and we can use spherical harmonics. As we will show, in this model it is also possible to use stochastic analysis.

2. Preliminaries. Consider $\mathbb{C}^{n}=\left\{z=\left(z_{1}, \ldots, z_{n}\right): z_{i} \in \mathbb{C}, i=\right.$ $1, \ldots, n\}$ with the Hermitian scalar product: for $z=\left(z_{1}, \ldots, z_{n}\right)$ and $w=$ $\left(w_{1}, \ldots, w_{n}\right)$,

$$
\langle z, w\rangle=\sum_{j=1}^{n} z_{j} \bar{w}_{j} .
$$

Then $|z|^{2}=\langle z, z\rangle=\sum_{j=1}^{n}\left|z_{j}\right|^{2}$.

A. Complex hyperbolic space and its isometries. Let $B_{1}=\left\{z \in \mathbb{C}^{n}\right.$ : $|z|<1\}$ denote the unit ball of $\mathbb{C}^{n}$. This set, equipped with the Bergman metric, is a model of a complex hyperbolic space. The Bergman metric is induced by the form $h=-4 \partial \bar{\partial} \log K(z)$ with $K(z)=1-|z|^{2}$ (cf. [Kr] or $[\mathrm{S}]$ ), which means that the metric is given by the matrix $\left[h_{i j}\right]$, where for $i, j=1, \ldots, n$,

$$
h_{i j}=\frac{\left(1-|z|^{2}\right) \delta_{i j}+\bar{z}_{i} z_{j}}{\left(1-|z|^{2}\right)^{2}} .
$$

Consider the Laplace-Beltrami operator associated to the Bergman metric on $B_{1}$ (we choose a normalization as in $[\mathrm{R}]$ )

$$
\Delta_{\mathrm{LB}}=4\left(1-|z|^{2}\right) \sum_{j, k=1}^{n}\left(\delta_{j k}-z_{j} \bar{z}_{k}\right) \frac{\partial^{2}}{\partial \bar{z}_{k} \partial z_{j}} .
$$


If $n=1$ then $\Delta_{\mathrm{LB}}=\left(1-|z|^{2}\right)^{2} \Delta$, where $\Delta$ is the ordinary Laplace operator, and the Bergman metric is the Poincaré metric. In this case the $\Delta_{\mathrm{LB}}$-harmonic functions coincide with the functions harmonic in the ordinary sense (see $[\mathrm{R}])$. Henceforth we assume $n \geq 2$.

All transformations of $B_{1}$ that are isometries of the Bergman metric are described in $[\mathrm{R}]$. Fix $a \in \mathbb{C}^{n}, a \neq 0$, and denote by $P_{a}$ the orthogonal projection of $\mathbb{C}^{n}$ on the complex line $\operatorname{lin}[a]$ and by $Q_{a}=I-P_{a}$ the orthogonal projection on the orthogonal complement to $\operatorname{lin}[a]$. More precisely, for $z \in \mathbb{C}^{n}$ put

$$
P_{a} z=\frac{\langle z, a\rangle}{\langle a, a\rangle} a \quad \text { and } \quad \psi_{a}(z)=\frac{a-P_{a} z-s_{a} Q_{a} z}{1-\langle z, a\rangle},
$$

where $s_{a}=\left(1-|a|^{2}\right)^{1 / 2}$. If $\psi$ is any isometry of $B_{1}$ and $a=\psi^{-1}(0)$, then there exists $g \in U(n)$ such that $\psi=g \psi_{a}$ (Theorem 2.2.5 in [R]).

B. Hypergeometric function. For $a, b, c \in \mathbb{C}, c \neq 0,-1,-2, \ldots$, and $|z|$ $<1$ the hypergeometric function ${ }_{2} F_{1}(a, b ; c ; z)$ is defined by the following power series $([\mathrm{E}])$ :

$$
{ }_{2} F_{1}(a, b ; c ; z)=\sum_{k=0}^{\infty} \frac{(a)_{k}(b)_{k}}{(c)_{k} k !} z^{k},
$$

where $(\alpha)_{0}=1$ and $(\alpha)_{k}=\alpha(\alpha+1) \cdots(\alpha+k-1)$ for $k=1,2, \ldots$ is the Pochhammer symbol. Observe that the roles of $a$ and $b$ are symmetric: ${ }_{2} F_{1}(a, b ; c ; z)={ }_{2} F_{1}(b, a ; c ; z)$. The function is also defined for $c=$ $0,-1,-2, \ldots$ provided $a$ (or $b$ ) is also a negative integer and $c \leq a<0$. Namely, if $a$ is a negative integer, $a=-m$, then for $c=-m-l$ and $l=0,1,2, \ldots$,

$$
{ }_{2} F_{1}(-m, b ;-m-l ; z)=\sum_{k=0}^{m} \frac{(-m)_{k}(b)_{k}}{(-m-l)_{k} k !} z^{k} .
$$

Let $n \geq 2$ be an integer and let $p, q$ be nonnegative integers. We will use the hypergeometic function for two sets of parameters: $a=p, b=q$, $c=p+q+n$, and $a=1-n-q, b=1-n-p, c=2-n-p-q$. In the first case the hypergeometric function is well defined for all $z \in \mathbb{C}$ with $|z| \leq 1$. In the second case it is a polynomial, provided $q>0$ (or $p>0$ ), because then $2-n-p-q<1-n-q$. Thus we have to define ${ }_{2} F_{1}(1-n-q, 1-n-p ; 2-n-p-q ; x)$ for $p=q=0$. In that case we additionally put $([\mathrm{E}])$

$$
{ }_{2} F_{1}(1-n, 1-n ; 2-n ; z)=\sum_{k=0}^{n-2} \frac{(-1)^{n-k}}{n-1-k}\left(\begin{array}{c}
n-1 \\
k
\end{array}\right) z^{k}+z^{n-1} \ln z
$$


If $\Re c>\Re b>0$ and $\Re(c-a-b)>0$, then the Gauss ${ }_{2} F_{1}$ identity states that

$$
{ }_{2} F_{1}(a, b ; c ; 1)=\frac{\Gamma(c) \Gamma(c-a-b)}{\Gamma(c-a) \Gamma(c-b)} .
$$

Moreover, if $c<b<a<0$ are integers, the series defining ${ }_{2} F_{1}(a, b ; c ; z)$ terminates and an analogue of the Gauss identity holds true (cf. [V]).

Differentiation of the hypergeometric function gives again the hypergeometric function

$$
\frac{d^{k}}{d x^{k}}{ }_{2} F_{1}(a, b ; c ; z)=\frac{(a)_{k}(b)_{k}}{(c)_{k}}{ }_{2} F_{1}(a+k, b+k ; c+k ; z)
$$

and one of Kummer's relations (see [E, 2.9]) gives the identity

$$
{ }_{2} F_{1}(a, b ; c ; z)=(1-z)^{c-a-b} F(c-a, c-b ; c ; z) .
$$

The function $y(z)={ }_{2} F_{1}(a, b ; c ; z)$ satisfies the hypergeometric equation

$$
z(1-z) y^{\prime \prime}(z)+(c-(a+b+1) z) y^{\prime}(z)-a b y(z)=0 .
$$

For $a=p, b=q, c=n+p+q$, the general solution of (1) is given by the formula $([\mathrm{E}])$

$$
y(z)=c_{1} \cdot{ }_{2} F_{1}(a, b ; c ; z)+\frac{c_{2}}{z^{1-c}} \cdot{ }_{2} F_{1}(1+a-c, 1+b-c ; 2-c ; z) .
$$

C. Jacobi polynomials. For $\alpha, \beta>-1$ and $k=0,1,2, \ldots$ the Jacobi polynomial of degree $k$ associated to $(\alpha, \beta)$ is given by

$$
P_{k}^{\alpha, \beta}(x)=\frac{(-1)^{k}(1-x)^{-\alpha}(1+x)^{-\beta}}{k ! 2^{k}} \frac{d^{k}}{d x^{k}}\left[(1-x)^{\alpha+k}(1+x)^{\beta+k}\right]
$$

with

$$
P_{k}^{\alpha, \beta}(1)=\frac{\Gamma(\alpha+k+1)}{k ! \Gamma(\alpha+1)}=\left(\begin{array}{c}
k+\alpha \\
k
\end{array}\right)
$$

The Jacobi polynomials satisfy the Jacobi equation: for $-1 \leq x \leq 1$,

$$
(1-x)^{2} y^{\prime \prime}(x)+[\beta-\alpha-(\alpha+\beta+2) x] y^{\prime}(x)+k(k+\alpha+\beta+1) y(x)=0 .
$$

For fixed $\alpha, \beta$ they form an orthogonal basis in $L^{2}\left([-1,1],(1-x)^{\alpha}(1+x)^{\beta} d x\right)$ :

$$
\begin{aligned}
\int_{-1}^{1} P_{k}^{\alpha, \beta}(x) P_{m}^{\alpha, \beta}(x) & (1-x)^{\alpha}(1+x)^{\beta} d x \\
& =\delta_{k m} \frac{2^{\alpha+\beta+1} \Gamma(\alpha+k+1) \Gamma(\beta+k+1)}{k !(k+\alpha+\beta+2 k+1) \Gamma(\alpha+\beta+k+1)} .
\end{aligned}
$$

D. Spherical harmonics in $\mathbb{C}^{n}$. We can identify the set $\mathbb{C}^{n}$ with $\mathbb{R}^{2 n}$. Then $S_{1}=\partial B_{1}$, the unit sphere in $\mathbb{C}^{n}$, coincides with the unit sphere in $\mathbb{R}^{2 n}$. Fix $n$ and let $\sigma_{1}$ denote the surface measure on the unit sphere in $\mathbb{R}^{2 n}$. We denote by $\omega_{2 n-1}=2 \pi^{n} / \Gamma(n)$ the area of the unit sphere in $\mathbb{R}^{2 n}$.

Consider the unitary group $U(n)$ acting on $S_{1}$. The subgroup of unitary transformations which leave one point of $S_{1}$ fixed is $U(n-1)$, and the sphere 
can be identified with the homogeneous space $U(n) / U(n-1)$. Functions on $S_{1}$ invariant under $U(n-1)$ are called zonal functions. It turns out $([\mathrm{K}]$, see also Proposition 1 in $[\mathrm{F}])$ that there exists an orthogonal decomposition of the function space $L^{2}\left(S_{1}, \sigma_{1}\right)$ into subspaces $\mathcal{H}^{p, q}, p, q=0,1,2, \ldots$, which are invariant and irreducible under $U(n)$. The functions belonging to $\mathcal{H}^{p, q}$ are called spherical harmonics. The zonal harmonics $H_{n}^{p, q} \in \mathcal{H}^{p, q}$ are the following: for $z=R e^{i \theta} \in \mathbb{C}$,

$$
H_{n}^{p, q}\left(R e^{i \theta}\right)=\frac{D(p, q, n)}{\omega_{2 n-1} P_{p \wedge q}^{n-2,|p-q|}(1)} e^{i(p-q) \theta} R^{|p-q|} P_{p \wedge q}^{n-2,|p-q|}\left(2 R^{2}-1\right),
$$

where $P_{p \wedge q}^{n-2,|p-q|}\left(2 R^{2}-1\right)$ is the appropriate Jacobi polynomial, $p \wedge q=$ $\min (p, q)$ and

$$
D(p, q, n)=\frac{(p+q+n-1)(p+n-2) !(q+n-2) !}{p ! q !(n-1) !(n-2) !}
$$

is the dimension of the space $\mathcal{H}^{p, q}$. Observe also that $\overline{H_{n}^{p, q}\left(\operatorname{Re} e^{i \theta}\right)}=H_{n}^{q, p}\left(R e^{i \theta}\right)$ and $H_{n}^{p, q}(1)=H_{n}^{q, p}(1)=D(p, q, n) \omega_{2 n-1}^{-1}$.

It is also well-known (cf. e.g. $[\mathrm{K}])$ that $\left(H_{n}^{p, q}\right)_{p, q=0}^{\infty}$ is an orthogonal basis in $L^{2}\left(\left\{(x, y) \in \mathbb{R}^{2}: x^{2}+y^{2}<1\right\},\left(1-x^{2}-y^{2}\right)^{n-2} d x d y\right)$.

The orthogonal projections from $L^{2}\left(S_{1}, \sigma_{1}\right)$ onto $\mathcal{H}^{p, q}$ were described by Koornwinder $[\mathrm{K}]$; the formulation below is taken from $[\mathrm{F}]$ :

Proposition 1. If $f_{1}, \ldots, f_{D(p, q, n)}$ is any orthonormal basis for $\mathcal{H}^{p, q} \subset$ $L^{2}\left(S_{1}, \sigma_{1}\right)$, then for $x, y \in S_{1}$,

$$
\sum_{j=1}^{D(p, q, n)} f_{j}(x) \overline{f_{j}(y)}=H_{n}^{p, q}(\langle x, y\rangle)
$$

Consequently, the orthogonal projection $\pi_{p, q}: L^{2}\left(S_{1}, \sigma_{1}\right) \rightarrow \mathcal{H}^{p, q}$ is given by

$$
\pi_{p, q} f(x)=\int_{S_{1}} H_{n}^{p, q}(\langle x, y\rangle) f(y) d \sigma_{1}(y) .
$$

Fix $x \in B_{1}, x \neq 0$, and let $a=R e^{i \theta}$ with $R \leq 1$. Put $S_{x, a}=\left\{z \in S_{1}:\right.$ $\langle z, x\rangle /(|z||x|)=a\}$. Observe that $S_{x, a}$ is a sphere in $\mathbb{C}^{n-1}$. In this way we decompose the unit sphere as $S_{1}=D \times S_{x, a}$, where $D$ is the unit disc in the complex plane.

If $g_{x} \in U(n)$ is such that $g_{x} x=x$, then of course $g_{x}\left(S_{x, a}\right)=S_{x, a}$. Let $\sigma_{x, a}$ denote the spherical measure on $S_{x, a} \subset \mathbb{R}^{2 n-2}$. The above decomposition of $S_{1}$ induces a decomposition of $\sigma_{1}:$ if $z=\left(a, z_{a}\right)$ with $a=R e^{i \theta}$ and $z_{a} \in S_{x, a}$, then

$$
d \sigma_{1}(z)=d \sigma_{x, a}\left(z_{a}\right) R\left(1-R^{2}\right)^{n-2} d R d \theta .
$$

Let $f_{x}$ be a function defined on $S_{1}$, continuous and invariant under those $g_{x} \in U(n)$ which leave $x$ fixed. Such functions $f_{x}$ are uniquely determined 
by their expansions with respect to $\left(H_{n}^{p, q}\right)_{p, q=0}^{\infty}$. Indeed, if in view of (4) we define the $(p, q)$-coefficient of $f_{x}$ as

$$
\left(\widehat{f_{x}}\right)_{p, q}=\int_{S_{1}} H_{n}^{p, q}(\langle x, z\rangle) f_{x}(z) d \sigma_{1}(z)=\int_{S_{1}} H_{n}^{q, p}(\langle z, x\rangle) f_{x}(z) d \sigma_{1}(z),
$$

then, by the above decomposition of the unit sphere, we have

$$
\begin{aligned}
\left(\widehat{f}_{x}\right)_{p, q} & =\int_{D}\left(\int_{S_{x, a}} H_{n}^{p, q}\left(\left\langle x, z_{a}\right\rangle\right) f_{x}\left(\left(a, z_{a}\right)\right) d \sigma_{x, a}\left(z_{a}\right)\right) R\left(1-R^{2}\right)^{n-2} d R d \theta \\
& =\int_{D} H_{n}^{p, q}\left(R e^{i \theta}\right) \widetilde{f}_{x}\left(R e^{i \theta}\right) R\left(1-R^{2}\right)^{n-2} d R d \theta
\end{aligned}
$$

where $\widetilde{f}_{x}\left(R e^{i \theta}\right)=\widetilde{f}_{x}(a)=f_{x}\left(a, z_{a}\right)$ for some (and hence all) $z_{a} \in S_{x, a}$. The family of harmonics $\left(H_{n}^{p, q}\left(R e^{i \theta}\right)\right)_{p, q=0,1,2 \ldots}$ is an orthogonal basis in $L^{2}\left(D, R\left(1-R^{2}\right)^{n-2} d R d \theta\right)$, hence the coefficients $\left(\widehat{f}_{x}\right)_{p, q}, p, q=0,1,2, \ldots$, uniquely determine the values of $f_{x}$ on every $S_{x, a}$, whence on the whole sphere $S_{1}$.

If $f_{x}$ is defined on the sphere $S_{r}=\left\{z \in \mathbb{C}^{n}:|z|=r\right\}$ and $\sigma_{r}$ denotes the spherical measure on $S_{r}$, then we define

$$
\begin{aligned}
\left(\widehat{f_{x}}\right)_{p, q} & =\int_{S_{r}} H_{n}^{p, q}\left(\frac{\langle x, z\rangle}{|x||z|}\right) f_{x}(z) d \sigma_{r}(z) \\
& =\int_{S_{r}} H_{n}^{q, p}\left(\frac{\langle z, x\rangle}{|z||x|}\right) f_{x}(z) d \sigma_{r}(z) .
\end{aligned}
$$

E. Diffusion: relation between its generator and a stochastic differential equation. The following fact is well-known. The proof can be found in many textbooks, e.g. [RW]. Let $\left(X_{t}\right)_{t \geq 0}$ be a real-valued diffusion with the following generator: for $f$ twice differentiable,

$$
A f(x)=\sigma(x) f^{\prime \prime}(x)+b(x) f^{\prime}(x),
$$

where $\sigma, b$ are continuous functions and $\sigma>0$ is locally Lipschitz. Then $\left(X_{t}\right)$ satisfies the stochastic differential equation

$$
d X_{t}=\sqrt{\sigma\left(X_{t}\right)} d W_{t}+b\left(X_{t}\right) d t,
$$

where $\left(W_{t}\right)_{t \geq 0}$ is a real-valued Brownian motion with $\mathbb{E}\left(W_{t}\right)=2 t$.

3. The process $Y_{t}=\left\langle X_{t}, a\right\rangle /\left(\left|X_{t}\right||a|\right)$ and its polar decomposition. Fix a nonzero point $a \in B_{1}$ and consider the isometry $\psi_{a}$ of $B_{1}$ equipped with the Bergman metric. From the description of such isometries in item A of Section 2 we know that, modulo unitary transformations, $\psi_{a}$ is a combination of two orthogonal projections. If we consider $\psi_{a}\left(X_{t}\right)$, it turns out that the 
projection

$$
P_{a}\left(X_{t}\right)=\frac{\left\langle X_{t}, a\right\rangle}{\langle a, a\rangle} a=\left\langle X_{t}, \frac{a}{|a|}\right\rangle \cdot \frac{a}{|a|}
$$

contains essential information about the behaviour of the process $\left(X_{t}\right)$.

More precisely: consider the complex hyperbolic Brownian motion $\left(X_{t}\right)_{t \geq 0}$ starting from the point $a \in B_{1}, a \neq 0$. With probability one, this process does not visit zero. Indeed, consider the projection of $X_{t}$ onto $\left\{z \in \mathbb{C}^{n}: z=\right.$ $\left.\left(z_{1}, 0, \ldots, 0\right)\right\}$. Computing the action of $\Delta_{\mathrm{LB}}$ on functions $f\left(z_{1}, 0, \ldots, 0\right)=$ $g\left(z_{1}\right)$, depending on $z_{1}$ only, we easily see that the generator of this twodimensional process is $\left(1-|z|^{2}\right)\left(1-\left|z_{1}\right|^{2}\right) \Delta_{\mathbb{R}^{2}}$, hence the process is a timechanged planar Brownian motion. With probability one, the planar Brownian motion does not visit zero, hence neither does $\left(X_{t}\right)_{t \geq 0}$. This implies that the process

$$
Y_{t}=\frac{\left\langle X_{t}, a\right\rangle}{\left|X_{t}\right||a|}
$$

is well-defined. Observe that $Y_{t}$ is a complex-valued process with values in the unit disc of the complex plane, hence we can decompose it using polar coordinates:

$$
Y_{t}=\frac{\left\langle X_{t}, a\right\rangle}{\left|X_{t}\right||a|}=R_{t} e^{i \theta_{t}}
$$

where

$$
R_{t}=\frac{\left|\left\langle X_{t}, a\right\rangle\right|}{\left|X_{t}\right||a|}
$$

is the length of the projection of the vector $X_{t} /\left|X_{t}\right|$ onto $\operatorname{lin}[a]$, and

$$
e^{i \theta_{t}}=\frac{\left\langle X_{t}, a\right\rangle}{\left|\left\langle X_{t}, a\right\rangle\right|}
$$

measures the angle (on the plane $\operatorname{lin}[a]$ ) between this projection and $a$.

4. The radial process $\left(r_{t}\right)_{t \geq 0}$ and its generator. We will need one more process, the length of $X_{t}$. As the norm is not differentiable at zero, instead of $\left|X_{t}\right|$ we will consider its square:

$$
r_{t}=\left|X_{t}\right|^{2}
$$

For $z \in \mathbb{C}^{n}$ put $r=|z|^{2}=\sum_{j=1}^{n} z_{j} \bar{z}_{j}$. To compute the generator of the process $\left(r_{t}\right)_{t \geq 0}$ it is enough to compute $\Delta_{\mathrm{LB}} f(r)$ for twice differentiable real functions $f$. Because

$$
\frac{\partial r}{\partial \bar{z}_{k}}=z_{k}, \quad \frac{\partial r}{\partial z_{j}}=\bar{z}_{j}, \quad \frac{\partial^{2} r}{\partial \bar{z}_{k} \partial z_{j}}=\delta_{j k},
$$

for any function $f:(0, \infty) \rightarrow \mathbb{R}$ with two continuous derivatives we have 
(8)

$$
\begin{array}{rl}
\Delta_{\mathrm{LB}} & f(r) \\
& =4\left(1-|z|^{2}\right) \cdot \sum_{j, k=1}^{n}\left(\delta_{j k}-z_{j} \bar{z}_{k}\right) \frac{\partial^{2} f(r)}{\partial \bar{z}_{k} \partial z_{j}} \\
& =4\left(1-|z|^{2}\right) \cdot \sum_{j, k=1}^{n}\left(\delta_{j k}-z_{j} \bar{z}_{k}\right)\left(f^{\prime \prime}(r) \frac{\partial r}{\partial \bar{z}_{k}} \cdot \frac{\partial r}{\partial z_{j}}+f^{\prime}(r) \frac{\partial^{2} r}{\partial \bar{z}_{k} \partial z_{j}}\right) \\
& =4 r(1-r)^{2} f^{\prime \prime}(r)+4(1-r)(n-r) f^{\prime}(r) .
\end{array}
$$

This implies that the process $\left(r_{t}\right)_{t \geq 0}$ is a solution of the stochastic differential equation

$$
d r_{t}=2 \sqrt{r_{t}}\left(1-r_{t}\right) d W_{t}^{(1)}+4\left(1-r_{t}\right)\left(n-r_{t}\right) d t
$$

where $\left(W_{t}^{(1)}\right)_{t \geq 0}$ is a real-valued Brownian motion normalized in such a way that $\mathbb{E}\left(W_{t}^{(1)}\right)=2 t$.

5. Generators of the processes $\left(R_{t}\right)_{t \geq 0}$ and $\left(\theta_{t}\right)_{t \geq 0}$. Fix $a \neq 0$ and put

$$
A=\langle a, z\rangle=\sum_{j=1}^{n} a_{j} \bar{z}_{j}, \quad \bar{A}=\langle z, a\rangle=\sum_{j=1}^{n} z_{j} \bar{a}_{j} .
$$

By the formulas (6) and (7) we have

$$
R=\frac{|\langle z, a\rangle|}{|z||a|}=\frac{|A|}{|a||z|} \quad \text { and } \quad e^{i \theta}=\frac{\langle z, a\rangle}{|\langle z, a\rangle|}=\frac{\langle z, a\rangle}{\sqrt{\langle a, z\rangle\langle z, a\rangle}}=\frac{\bar{A}}{\sqrt{A \bar{A}}},
$$

hence $\theta=-i \ln (\bar{A} / \sqrt{A \bar{A}})$, where $\ln z$ denotes the principal branch of the $\operatorname{logarithm}$, i.e. the continuous branch with $\ln 1=0$.

We will need the partial derivatives of these functions:

$$
\begin{gathered}
\frac{\partial \theta}{\partial \bar{z}_{k}}=\frac{i a_{k}}{2 A}, \quad \frac{\partial \theta}{\partial z_{j}}=\frac{-i \bar{a}_{j}}{2 \bar{A}}, \quad \frac{\partial^{2} \theta}{\partial \bar{z}_{k} \partial z_{j}}=0 \\
\frac{\partial R}{\partial \bar{z}_{k}}=\frac{a_{k} \bar{A}}{2|a||z||A|}-\frac{z_{k}|A|}{2|a||z|^{3}}, \quad \frac{\partial R}{\partial z_{j}}=\frac{\bar{a}_{j} A}{2|a||z||A|}-\frac{\bar{z}_{j}|A|}{2|a||z|^{3}}
\end{gathered}
$$

and

$$
\begin{aligned}
\frac{\partial^{2} R}{\partial \bar{z}_{k} \partial z_{j}}= & \frac{a_{k} \bar{a}_{j}}{4|a||z||A|}-\frac{a_{k} \bar{z}_{j} \bar{A}}{4|a||z|^{3}|A|} \\
& -\frac{\delta_{j k}|A|}{2|a||z|^{3}}-\frac{\bar{a}_{j} z_{k} A}{4|a||z|^{3}|A|}+\frac{3 z_{k} \bar{z}_{j}|A|}{4|a||z|^{5}} .
\end{aligned}
$$

Let $f$ be a twice differentiable real function. We compute the action of $\Delta_{\mathrm{LB}}$ on $f(R)$ : 
(13)

$$
\begin{array}{rl}
\Delta_{\mathrm{LB}} & f(R) \\
& =4\left(1-|z|^{2}\right) \sum_{j, k=1}^{n}\left(\delta_{j k}-z_{j} \bar{z}_{k}\right) \frac{\partial^{2} f(R)}{\partial \bar{z}_{k} \partial z_{j}} \\
& =4\left(1-|z|^{2}\right) \sum_{j, k=1}^{n}\left(\delta_{j k}-z_{j} \bar{z}_{k}\right)\left(f^{\prime \prime}(R) \frac{\partial R}{\partial \bar{z}_{k}} \frac{\partial R}{\partial z_{j}}+f^{\prime}(R) \frac{\partial^{2} R}{\partial \bar{z}_{k} \partial z_{j}}\right) .
\end{array}
$$

Using (11), we get

$$
\begin{aligned}
4\left(1-|z|^{2}\right) & \sum_{j, k=1}^{n}\left(\delta_{j k}-z_{j} \bar{z}_{k}\right) \frac{\partial R}{\partial \bar{z}_{k}} \frac{\partial R}{\partial z_{j}} \\
= & 4\left(1-|z|^{2}\right) \sum_{j, k=1}^{n}\left(\delta_{j k}-z_{j} \bar{z}_{k}\right)\left(\frac{a_{k} \bar{A}}{2|a||z||A|}-\frac{z_{k}|A|}{2|a||z|^{3}}\right) \\
& \times\left(\frac{\bar{a}_{j} A}{2|a||z||A|}-\frac{\bar{z}_{j}|A|}{2|a||z|^{3}}\right) \\
= & \frac{1-|z|^{2}}{|z|^{2}}\left(1-\frac{|A|^{2}}{|a|^{2}|z|^{2}}\right)=\frac{1-|z|^{2}}{|z|^{2}}\left(1-R^{2}\right)
\end{aligned}
$$

and using (12) we obtain

$$
\begin{aligned}
4\left(1-|z|^{2}\right) & \sum_{j, k=1}^{n}\left(\delta_{j k}-z_{j} \bar{z}_{k}\right) \frac{\partial^{2} R}{\partial \bar{z}_{k} \partial z_{j}} \\
= & 4\left(1-|z|^{2}\right) \sum_{j, k=1}^{n}\left(\delta_{j k}-z_{j} \bar{z}_{k}\right)\left(\frac{a_{k} \bar{a}_{j}}{4|a||z||A|}-\frac{a_{k} \bar{z}_{j} \bar{A}}{4|a||z|^{3}|A|}\right. \\
& \left.-\frac{\delta_{j k}|A|}{2|a||z|^{3}}-\frac{\bar{a}_{j} z_{k} A}{4|a||z|^{3}|A|}+\frac{3 z_{k} \bar{z}_{j}|A|}{4|a||z|^{5}}\right) \\
= & \frac{1-|z|^{2}}{|z|^{2}}\left(\frac{1}{R}-(2 n-1) R\right)
\end{aligned}
$$

hence

$$
\Delta_{\mathrm{LB}} f(R)=\frac{1-|z|^{2}}{|z|^{2}}\left(\left(1-R^{2}\right) f^{\prime \prime}(R)+\left(\frac{1}{R}-(2 n-1) R\right) f^{\prime}(R)\right) .
$$

This means that the real-valued process $R_{t}$ is a diffusion, described by the stochastic equation

$$
\left.d R_{t}=\sqrt{\frac{1-r_{t}}{r_{t}}} \sqrt{1-R_{t}^{2}} d W_{t}^{(2)}+\frac{1-r_{t}}{r_{t}}\left(\frac{1}{R_{t}}-(2 n-1) R_{t}\right)\right) d t .
$$

In a similar way, computing the action of $\Delta_{\mathrm{LB}}$ on $g(\theta)$ and using (10), we find that 


$$
\Delta_{\mathrm{LB}} g(\theta)=\frac{1-|z|^{2}}{|z|^{2}}\left(\frac{1}{R^{2}}-|z|^{2}\right) g^{\prime \prime}(\theta)
$$

hence

$$
d \theta_{t}=\sqrt{\frac{1-r_{t}}{r_{t}}} \sqrt{\frac{1}{R_{t}^{2}}-r_{t}} d W_{t}^{(3)}
$$

6. Independence of $W^{(1)}, W^{(2)}$ and $W^{(3)}$. Now we will show that the Brownian motions $W_{t}^{(1)}, W_{t}^{(2)}$ and $W_{t}^{(3)}$ can be chosen to be independent. We are interested in the martingale parts of the processes $r_{t}, R_{t}$ and $\theta_{t}$; for convenience denote them by superscript $\mathrm{m}$. For instance, $\left(r_{t}\right)^{\mathrm{m}}$ will denote the martingale part of $r_{t}$, i.e. the stochastic integral $\int_{0}^{t} 2 \sqrt{r_{s}}\left(1-r_{s}\right) d W_{s}^{(1)}$.

The group $U(n)$ acts transitively on the unit sphere, so we may assume that $a=\left(a_{1}, 0, \ldots, 0\right)$. Then $(6),(7)$ and the definition of $r_{t}$ imply that $X_{1}(t)$, the first coordinate of the hyperbolic Brownian motion $X(t)$, is $\sqrt{r_{t}} R_{t} e^{i \theta_{t}}$. First we will show the independence of $W_{t}^{(1)}$ and $W_{t}^{(2)}$, the Brownian motions from formulas (9) and (17).

On the one hand, as shown in Section 3, the process $X_{1}(t)$ has the generator

$$
(1-r)\left(1-R^{2} r\right) \Delta_{\mathbb{R}^{2}}
$$

This implies that the process $\left|X_{1}(t)\right|$, being a time-changed Bessel process of dimension two, has the following bracket:

$$
\left\langle\left|X_{1}\right|,\left|X_{1}\right|\right\rangle_{t}=2\left(1-r_{t}\right)\left(1-R_{t}^{2} r_{t}\right) .
$$

On the other hand, $\left|X_{1}(t)\right|=\sqrt{r_{t}} R_{t}$, thus (9) and (17) give another way to compute the bracket of $\left|X_{1}(t)\right|$. By the Itô formula,

$$
d\left(\left|X_{1}(t)\right|\right)^{\mathrm{m}}=R_{t} d\left(\sqrt{r_{t}}\right)^{\mathrm{m}}+\sqrt{r_{t}} d\left(R_{t}\right)^{\mathrm{m}},
$$

hence first we have to find the stochastic equation describing the martingale part of the process $\sqrt{r_{t}}$. We make use of the Itô formula once again to get $d\left(\sqrt{r_{t}}\right)^{\mathrm{m}}=\left(1-r_{t}\right) d W_{t}^{(1)}$. From this and (17), we get

$$
\begin{aligned}
d\left(\left|X_{1}(t)\right|\right) & =R_{t}\left(1-r_{t}\right) d W_{t}^{(1)}+\sqrt{r_{t}} d\left(R_{t}\right)^{\mathrm{m}} \\
& =R_{t}\left(1-r_{t}\right) d W_{t}^{(1)}+\sqrt{1-r_{t}} \sqrt{1-R_{t}^{2}} d W_{t}^{(2)} .
\end{aligned}
$$

We can now compute the bracket of $\left|X_{1}(t)\right|$ :

$$
\begin{aligned}
\left\langle\left|X_{1}\right|,\left|X_{1}\right|\right\rangle_{t}= & 2\left(R_{t}^{2}\left(1-r_{t}\right)^{2}+\left(\sqrt{1-r_{t}}\right)^{2}\left(\sqrt{1-R_{t}^{2}}\right)^{2}\right) \\
& +R_{t} \sqrt{1-R_{t}^{2}}\left(\sqrt{1-r_{t}}\right)^{3}\left\langle W^{(1)}, W^{(2)}\right\rangle_{t} \\
= & 2\left(1-r_{t}\right)\left(1-r_{t} R_{t}^{2}\right)+R_{t} \sqrt{1-R_{t}^{2}}\left(\sqrt{1-r_{t}}\right)^{3}\left\langle W^{(1)}, W^{(2)}\right\rangle_{t} .
\end{aligned}
$$


Comparing this with (21) we see that $\left\langle W^{(1)}, W^{(2)}\right\rangle_{t}=0$, hence the Brownian motions $W^{(1)}$ and $W^{(2)}$ are independent.

We know that $X_{1}(t)=\sqrt{r_{t}} R_{t} e^{i \theta_{t}}$ and $X_{1}$ is a two-dimensional Brownian motion with random clock, because its generator, given in (20), is the Laplace operator in $\mathbb{R}^{2}$, multiplied by a function.

Let $(u, \theta)$ denote the polar coordinates on the plane, $0 \leq u<\infty, 0 \leq$ $\theta<2 \pi$. The polar decompositon of the Laplace operator on the plane,

$$
\Delta_{\mathbb{R}^{2}}=\frac{\partial^{2}}{\partial u^{2}}+\frac{1}{u} \frac{\partial}{\partial u}+\frac{1}{u^{2}} \frac{\partial^{2}}{\partial \theta^{2}}
$$

implies a representation of $X_{1}(t)$ as a skew-product of a radial part (which is a Bessel process) and an angular (spherical) part (cf. [PR] or [IMcK, Chapter 7.15]). More precisely, if $\left(B_{t}^{(1)}, B_{t}^{(2)}\right)$ is the standard two-dimensional Brownian motion then there exist a Bessel process $\operatorname{Bes}(t)$ and a real Brownian motion $W(t)$, independent of $\operatorname{Bes}(t)$, such that

$$
\left(B_{t}^{(1)}, B_{t}^{(2)}\right) \stackrel{d}{=}\left[\operatorname{Bes}(t), e^{i W(A(t))}\right],
$$

where $A(t)=\int_{0}^{t} \operatorname{Bes}^{-2}(s) d s$. The same applies in our situation, because our process $X_{1}(t)$ is a time-changed two-dimensional Brownian motion.

As we have already described the process $\sqrt{r_{t}} R_{t}$, which is the radial part of $X_{1}(t)$, we now focus on the spherical part. In our case $u=R \sqrt{r}$, hence the spherical part of $(20)$ is

$$
(1-r)\left(1-R^{2} r\right) \frac{1}{R^{2} r} \frac{\partial^{2}}{\partial \theta^{2}}=\frac{1-r}{r}\left(\frac{1}{R^{2}}-r\right) \frac{\partial^{2}}{\partial \theta^{2}}
$$

which gives the following representation for the process $e^{i \theta_{t}}$ : there exists a real Brownian motion $W^{(3)}$, independent of $W^{(1)}$ and $W^{(2)}$, such that

$$
d \theta_{t}=\sqrt{\frac{1-r_{t}}{r_{t}}} \sqrt{\frac{1}{R_{t}^{2}}-r_{t}} d W_{t}^{(3)}
$$

This is precisely equation (19), computed there by use of the generator.

REMARK. Independence of $W^{(1)}, W^{(2)}$ and $W^{(3)}$ can also be obtained by computing the action of $\Delta_{\mathrm{LB}}$ on the product $f(r) g(R) h(\theta)$, but this requires extensive computations.

7. A martingale. Our main tools in computing the Poisson kernel and Green function of the balls $B_{r}, 0<r<1$, are unitary spherical harmonics and killing $X_{t}$ when it reaches the boundary of $B_{r}$. In order to use unitary spherical harmonics we must have a complex-valued process; by analogy to the real case we consider $H_{n}^{q, p}\left(\left\langle X_{t}, a\right\rangle /\left(\left|X_{t}\right||a|\right)\right)$. For this reason in this 
section we examine the properties of the process

$$
Z_{t}=H_{n}^{q, p}\left(\frac{\left\langle X_{t}, a\right\rangle}{\left|X_{t}\right||a|}\right) \cdot \exp \left(\int_{0}^{t} Q\left(r_{s}\right) d s\right)
$$

where $\left(X_{t}\right)_{t \geq 0}$ is the complex hyperbolic Brownian motion, $a=X_{0} \neq 0$ is its starting point and $Q(r)$ is a function to be defined later.

We will find a positive, continuous function $Q\left(|z|^{2}\right)$ (a potential in the sense of [ChZ]) such that

$$
\left(\Delta_{\mathrm{LB}}-Q\left(|z|^{2}\right)\right)\left(H_{n}^{q, p}\left(\frac{\langle z, a\rangle}{|z||a|}\right)\right)=0 .
$$

It turns out (see e.g. [W, III.39, p. 159]) that for such a $Q$ the process $Z_{t}$ is a martingale.

Finding $Q$ by analytical methods requires tedious computations so we prefer using stochastic calculus and the Itô formula, which makes the calculations a little shorter.

Let $z=R e^{i \theta}$ be a complex number with modulus $R \leq 1$. Recall that by (3),

$$
H_{n}^{p, q}\left(R e^{i \theta}\right)=C e^{i(p-q) \theta} R^{|p-q|} P\left(2 R^{2}-1\right),
$$

where $C$ is some constant and $P=P_{p \wedge q}^{n-2,|p-q|}$ denotes the appropriate Jacobi polynomial. This implies that $H_{n}^{q, p}\left(R e^{i \theta}\right)=C e^{i(q-p) \theta} R^{|p-q|} P\left(2 R^{2}-1\right)=$ $\overline{H_{n}^{p, q}\left(R e^{i \theta}\right)}$.

For $n=2,3, \ldots$ and $p, q=0,1,2, \ldots$ consider the process

$$
Z_{t}=C e^{i(q-p) \theta_{t}} \cdot R_{t}^{|p-q|} P\left(2 R_{t}^{2}-1\right) \cdot \exp \left(\int_{0}^{t} Q\left(r_{s}\right) d s\right) .
$$

To simplify notation, write

$$
g\left(\theta_{t}\right)=C e^{i(q-p) \theta_{t}}, \quad f\left(R_{t}\right)=R_{t}^{|p-q|} P\left(2 R_{t}^{2}-1\right), \quad V_{t}=\exp \left(\int_{0}^{t} Q\left(r_{s}\right) d s\right) .
$$

Then

$$
Z_{t}=g\left(\theta_{t}\right) f\left(R_{t}\right) V_{t}, \quad Z_{0}=g(0) f(1) V_{0}=H_{n}^{p, q}(1)=D(p, q, n) \omega_{2 n-1}^{-1}
$$

and we have the following.

Theorem 2. Set

$$
Q(r)=4(1-r)\left[\frac{\frac{p+q}{2}\left(\frac{p+q}{2}+n-1\right)}{r}-\left(\frac{p-q}{2}\right)^{2}\right] .
$$


Then

$$
\begin{aligned}
d Z_{t}= & V_{t}\left\{g\left(\theta_{t}\right) f^{\prime}\left(R_{t}\right) \sqrt{\frac{1-r_{t}}{r_{t}}} \sqrt{1-R_{t}^{2}} d W_{t}^{(2)}\right. \\
& \left.+f\left(R_{t}\right) g^{\prime}\left(\theta_{t}\right) \sqrt{\frac{1-r_{t}}{r_{t}}} \sqrt{\frac{1}{R_{t}^{2}}-r_{t}} d W_{t}^{(3)}\right\},
\end{aligned}
$$

hence $\left(Z_{t}\right)_{t \geq 0}$ is a martingale and

$$
\begin{aligned}
Z_{t}= & H_{n}^{p, q}(1)+\int_{0}^{t} V_{t} g\left(\theta_{t}\right) f^{\prime}\left(R_{t}\right) \sqrt{\frac{1-r_{t}}{r_{t}}} \sqrt{1-R_{t}^{2}} d W_{s}^{(2)} \\
& +\int_{0}^{t} V_{t} f\left(R_{t}\right) g^{\prime}\left(\theta_{t}\right) \sqrt{\frac{1-r_{t}}{r_{t}}} \sqrt{\frac{1}{R_{t}^{2}}-r_{t}} d W_{s}^{(3)} .
\end{aligned}
$$

Proof. Observe that $Q$ is always positive, because $r \in(0,1)$ and $n \geq 2$. Below we use the Itô formula several times. Because $V_{t}$ is a process of bounded variation, we have $d V_{t}=Q\left(r_{t}\right) V_{t} d t$ and $\left\langle g\left(\theta_{t}\right) f\left(R_{t}\right), V_{t}\right\rangle=0$. Thus, differentiating $Z_{t}$ given by (23), we obtain

$$
d Z_{t}=d\left(g\left(\theta_{t}\right) f\left(R_{t}\right) V_{t}\right)=d\left(g\left(\theta_{t}\right) f\left(R_{t}\right)\right) \cdot V_{t}+g\left(\theta_{t}\right) f\left(R_{t}\right) \cdot d V_{t} .
$$

Now

$$
d\left(g\left(\theta_{t}\right) f\left(R_{t}\right)\right)=d\left(g\left(\theta_{t}\right)\right) f\left(R_{t}\right)+d\left(f\left(R_{t}\right)\right) g\left(\theta_{t}\right)+d\left\langle g\left(\theta_{t}\right), f\left(R_{t}\right)\right\rangle .
$$

By the Itô formula and (17),

$$
\begin{aligned}
& d\left(f\left(R_{t}\right)\right)=f^{\prime}\left(R_{t}\right) d R_{t}+\frac{1}{2} f^{\prime \prime}\left(R_{t}\right) d\left\langle R_{t}, R_{t}\right\rangle \\
& =f^{\prime}\left(R_{t}\right)\left[\sqrt{\frac{1-r_{t}}{r_{t}}} \sqrt{1-R_{t}^{2}} d W_{t}^{(2)}+\frac{1-r_{t}}{r_{t}}\left(\frac{1}{R_{t}}-(2 n-1) R_{t}\right) d t\right] \\
& \quad+f^{\prime \prime}\left(R_{t}\right)\left[\frac{1-r_{t}}{r_{t}}\left(1-R_{t}^{2}\right)\right] d t,
\end{aligned}
$$

because $d\left\langle W_{t}^{(2)}, W_{t}^{(2)}\right\rangle=2 t d t$.

In a similar way, using (19), we get

$$
\begin{aligned}
& d\left(g\left(\theta_{t}\right)\right)=g^{\prime}\left(\theta_{t}\right) d \theta_{t}+\frac{1}{2} g^{\prime \prime}\left(\theta_{t}\right) d\left\langle\theta_{t}, \theta_{t}\right\rangle \\
& \quad=g^{\prime}\left(\theta_{t}\right) \sqrt{\frac{1-r_{t}}{r_{t}}} \sqrt{\frac{1}{R_{t}^{2}}-r_{t}} d W_{t}^{(3)}+g^{\prime \prime}\left(\theta_{t}\right) \cdot \frac{1-r_{t}}{r_{t}} \cdot\left(\frac{1}{R_{t}^{2}}-r_{t}\right) d t
\end{aligned}
$$

Both $g\left(\theta_{t}\right)$ and $f\left(R_{t}\right)$ are continuous semimartingales, so that

$$
\left\langle g\left(\theta_{t}\right), f\left(R_{t}\right)\right\rangle=\left\langle g^{\mathrm{m}}\left(\theta_{t}\right), f^{\mathrm{m}}\left(R_{t}\right)\right\rangle,
$$

where the superscript " $\mathrm{m}$ " denotes the martingale part of the process ([RW, p. 58]). 
Moreover, because $W^{(2)}$ and $W^{(3)}$ in (17) and (19) are independent, we have $\left\langle g\left(\theta_{t}\right), f\left(R_{t}\right)\right\rangle=0$.

Substituting (27) and (28) into (26) and next (26) to (25), we get

$$
\begin{aligned}
= & V_{t}\left\{f\left(R_{t}\right) g^{\prime}\left(\theta_{t}\right) \sqrt{\frac{1-r_{t}}{r_{t}}} \sqrt{\frac{1}{R_{t}^{2}}-r_{t}} d W_{t}^{(3)}\right. \\
& +f\left(R_{t}\right) g^{\prime \prime}\left(\theta_{t}\right) \cdot \frac{1-r_{t}}{r_{t}} \cdot\left(\frac{1}{R_{t}^{2}}-r_{t}\right) d t \\
& +g\left(\theta_{t}\right) f^{\prime}\left(R_{t}\right)\left[\sqrt{\frac{1-r_{t}}{r_{t}}} \sqrt{1-R_{t}^{2}} d W_{t}^{(2)}+\frac{1-r_{t}}{r_{t}}\left(\frac{1}{R_{t}}-(2 n-1) R_{t}\right) d t\right] \\
& \left.+g\left(\theta_{t}\right) f^{\prime \prime}\left(R_{t}\right)\left[\frac{1-r_{t}}{r_{t}}\left(1-R_{t}^{2}\right)\right] d t+g\left(\theta_{t}\right) f\left(R_{t}\right) Q\left(r_{t}\right) d t\right\} .
\end{aligned}
$$

The right-hand side of (29) is a sum of two martingales,

$$
\begin{aligned}
& d M_{t}^{(1)}=V_{t} f\left(R_{t}\right) g^{\prime}\left(\theta_{t}\right) \sqrt{\frac{1-r_{t}}{r_{t}}} \sqrt{\frac{1}{R_{t}^{2}}-r_{t}} d W_{t}^{(3)}, \\
& d M_{t}^{(2)}=V_{t} g\left(\theta_{t}\right) f^{\prime}\left(R_{t}\right) \sqrt{\frac{1-r_{t}}{r_{t}}} \sqrt{1-R_{t}^{2}} d W_{t}^{(2)},
\end{aligned}
$$

and the following quantity:

$$
\begin{aligned}
V_{t}\{ & f\left(R_{t}\right) g^{\prime \prime}\left(\theta_{t}\right) \frac{1-r_{t}}{r_{t}}\left(\frac{1}{R_{t}^{2}}-r_{t}\right) \\
& +g\left(\theta_{t}\right) f^{\prime}\left(R_{t}\right) \frac{1-r_{t}}{r_{t}}\left(\frac{1}{R_{t}}-(2 n-1) R_{t}\right) \\
& \left.+g\left(\theta_{t}\right) f^{\prime \prime}\left(R_{t}\right) \frac{1-r_{t}}{r_{t}}\left(1-R_{t}^{2}\right)+g\left(\theta_{t}\right) f\left(R_{t}\right) Q\left(r_{t}\right)\right\} d t=V_{t} \cdot J_{t} d t
\end{aligned}
$$

Observe now that

$$
g^{\prime \prime}(\theta)=\left(C e^{i(q-p) \theta}\right)^{\prime \prime}=-C(q-p)^{2} e^{i(q-p) \theta}=-(p-q)^{2} g(\theta),
$$

hence $J_{t}$, the quantity in braces in (31), is equal to

$$
\begin{aligned}
J_{t}= & \frac{1-r_{t}}{r_{t}} g(\theta)\left[\left(1-R_{t}^{2}\right) f^{\prime \prime}\left(R_{t}\right)+\left(\frac{1}{R_{t}}-(2 n-1) R_{t}\right) f^{\prime}\left(R_{t}\right)\right. \\
& \left.-(p-q)^{2}\left(\frac{1}{R_{t}^{2}}-r_{t}\right) f\left(R_{t}\right)+\frac{r_{t}}{1-r_{t}} Q\left(r_{t}\right) f\left(R_{t}\right)\right] .
\end{aligned}
$$

But $f(R)=R^{|p-q|} P\left(2 R^{2}-1\right)$, hence

$$
f^{\prime}(R)=|p-q| R^{|p-q|-1} P\left(2 R^{2}-1\right)+4 R^{|p-q|+1} P^{\prime}\left(2 R^{2}-1\right),
$$




$$
\begin{aligned}
f^{\prime \prime}(R)= & |p-q|(|p-q|-1) R^{|p-q|-2} P\left(2 R^{2}-1\right) \\
& +(8|p-q|+4) R^{|p-q|} P^{\prime}\left(2 R^{2}-1\right)+16 R^{|p-q|+2} P^{\prime \prime}\left(2 R^{2}-1\right) .
\end{aligned}
$$

We want to find $Q$ that makes $J_{t}=0$. Substituting (33) and (34) into (32) we get a quantity which turns into the left-hand side of (2) if we put

$$
Q\left(r_{t}\right)=4\left(1-r_{t}\right)\left[\frac{\frac{p+q}{2}\left(\frac{p+q}{2}+n-1\right)}{r_{t}}-\left(\frac{p-q}{2}\right)^{2}\right] .
$$

Indeed,

$$
\begin{aligned}
J_{t}= & \frac{1-r_{t}}{r_{t}} g\left(\theta_{t}\right) R_{t}^{|p-q|}\left\{16\left(R_{t}^{2}-R_{t}^{4}\right) P^{\prime \prime}\left(2 R_{t}^{2}-1\right)\right. \\
& +8\left[1+|p-q|-(|p-q|+n) R_{t}^{2}\right] P^{\prime}\left(2 R_{t}^{2}-1\right) \\
& +\left[-|p-q|^{2}-(2 n-2)|p-q|+(p-q)^{2} r_{t}\right. \\
& \left.\left.+4 r_{t}\left(\frac{\frac{p+q}{2}\left(\frac{p+q}{2}+n-1\right)}{r_{t}}-\left(\frac{p-q}{2}\right)^{2}\right)\right] P\left(2 R_{t}^{2}-1\right)\right\} \\
= & 4 \frac{1-r_{t}}{r_{t}} g\left(\theta_{t}\right) R_{t}^{|p-q|}\left\{4\left(R_{t}^{2}-R_{t}^{4}\right) P^{\prime \prime}\left(2 R_{t}^{2}-1\right)\right. \\
& +2\left[|p-q|+1-(|p-q|+n) R_{t}^{2}\right] P^{\prime}\left(2 R_{t}^{2}-1\right) \\
& \left.+[p q+(n-1) \min (p, q)] P\left(2 R_{t}^{2}-1\right)\right\} .
\end{aligned}
$$

Substituting $x=2 R^{2}-1$ in the last braces above, we get

$$
\begin{aligned}
\left(1-x^{2}\right) P^{\prime \prime}(x)+[|p-q| & -n+2-(|p-q|+n) x] P^{\prime}(x) \\
& +[\min (p, q)(\min (p, q)+n-1+|p-q|)] P(x) .
\end{aligned}
$$

But this is precisely the left-hand side of (2), with $y(x)$ being the Jacobi polynomial $P(x)=P_{p \wedge q}^{n-2,|p-q|}(x)$. This implies that $J_{t}=0$, hence the quantity (31) is zero,

$$
\begin{aligned}
d Z_{t}= & V_{t} f\left(R_{t}\right) g^{\prime}\left(\theta_{t}\right) \sqrt{\frac{1-r_{t}}{r_{t}}} \sqrt{\frac{1}{R_{t}^{2}}-r_{t}} d W_{t}^{(3)} \\
& +V_{t} g\left(\theta_{t}\right) f^{\prime}\left(R_{t}\right) \sqrt{\frac{1-r_{t}}{r_{t}}} \sqrt{1-R_{t}^{2}} d W_{t}^{(2)}
\end{aligned}
$$

is a martingale and $Z_{0}=H_{n}^{q, p}\left(\left\langle X_{0}, a\right\rangle /\left(\left|X_{0}\right||a|\right)\right)=H_{n}^{q, p}(1)$, because $X_{0}=a$.

8. Expectation of $H_{n}^{q, p}\left(\left\langle X_{t}, x\right\rangle /\left(\left|X_{t}\right||x|\right)\right)$. Fix $0<r<1$ and let $B_{r}=$ $\left\{z \in \mathbb{C}^{n}:|z|<r\right\}$. Let $\left(X_{t}\right)_{t \geq 0}$ be the complex hyperbolic Brownian motion with $X_{0}=x \neq 0$. Recall that $r_{t}=\left|X_{t}\right|^{2}$ and $Z_{t}=H_{n}^{q, p}\left(\left\langle X_{t}, x\right\rangle /\left(\left|X_{t}\right||x|\right)\right) V_{t}$. Let $h: \mathbb{R} \rightarrow \mathbb{R}$ be Borel measurable and bounded. 
THEOREM 3. Under the above assumptions,

$$
\mathbb{E}^{x}\left[h\left(\left|X_{t}\right|^{2}\right) H_{n}^{q, p}\left(\frac{\left\langle X_{t}, x\right\rangle}{\left|X_{t}\right||x|}\right)\right]=H_{n}^{q, p}(1) \cdot \mathbb{E}^{|x|^{2}}\left[h\left(r_{t}\right) \exp \left(-\int_{0}^{t} Q\left(r_{s}\right) d s\right)\right] .
$$

Proof. The process $Z_{t}$ is a martingale, hence its expectation is constant. In order to get $Z_{t}$ we multiply $H_{n}^{q, p}\left(\left\langle X_{t}, x\right\rangle /\left(\left|X_{t}\right||x|\right)\right)$ by $1=V_{t} V_{t}^{-1}$ :

$$
\begin{aligned}
\mathbb{E}^{x}\left[h\left(\left|X_{t}\right|^{2}\right) H_{n}^{q, p}\right. & \left.\left(\frac{\left\langle X_{t}, x\right\rangle}{\left|X_{t}\right||x|}\right)\right]=\mathbb{E}^{x}\left[h\left(r_{t}\right) H_{n}^{q, p}\left(\frac{\left\langle X_{t}, x\right\rangle}{\left|X_{t}\right||x|}\right) V_{t} V_{t}^{-1}\right] \\
& =\mathbb{E}^{x}\left[h\left(r_{t}\right) Z_{t} V_{t}^{-1}\right]=\mathbb{E}^{x}\left[h\left(r_{t}\right) Z_{t} \exp \left(-\int_{0}^{t} Q\left(r_{s}\right) d s\right)\right] .
\end{aligned}
$$

But we know the structure of $Z_{t}$ : by $(24)$ we have

$$
\begin{aligned}
\mathbb{E}^{x}\left[h\left(r_{t}\right) Z_{t} \exp \right. & \left.\left(-\int_{0}^{t} Q\left(r_{s}\right) d s\right)\right]=\mathbb{E}^{x}\left[h\left(r_{t}\right) V_{t}^{-1} H_{n}^{q, p}(1)\right] \\
& +\mathbb{E}^{x}\left[h\left(r_{t}\right) V_{t}^{-1}\left(\int_{0}^{t} V_{s} g\left(\theta_{s}\right) f^{\prime}\left(R_{s}\right) \sqrt{\frac{1-r_{s}}{r_{s}}} \sqrt{1-R_{s}^{2}} d W_{s}^{(2)}\right)\right] \\
& +\mathbb{E}^{x}\left[h\left(r_{t}\right) V_{t}^{-1}\left(\int_{0}^{t} V_{s} f\left(R_{s}\right) g^{\prime}\left(\theta_{s}\right) \sqrt{\frac{1-r_{s}}{r_{s}}} \sqrt{\frac{1}{R_{s}^{2}}-r_{s}} d W_{s}^{(3)}\right)\right]
\end{aligned}
$$

where $V_{t}^{-1}=\exp \left(-\int_{0}^{t} Q\left(r_{s}\right) d s\right)$. The process $h\left(r_{t}\right) \exp \left(-\int_{0}^{t} Q\left(r_{s}\right) d s\right)$ depends only on $\left(r_{s}\right)_{0 \leq s \leq t}$, hence it is measurable with respect to the filtration of the Brownian motion $W^{(1)}$. The stochastic integrals in the last two terms of the right-hand side are integrals with respect to the Brownian motions $W^{(2)}$ and $W^{(3)}$, respectively, and these Brownian motions are independent of $W^{(1)}$. Hence the last two terms in the above sum are zero (cf. also Lemma 2.4 in $[\mathrm{BM}])$ and we finally have

$$
\mathbb{E}^{x}\left[h\left(r_{t}\right) H_{n}^{q, p}\left(\frac{\left\langle X_{t}, x\right\rangle}{\left|X_{t}\right||x|}\right)\right]=H_{n}^{q, p}(1) \mathbb{E}^{|x|^{2}}\left[h\left(r_{t}\right) \exp \left(-\int_{0}^{t} Q\left(r_{s}\right) d s\right)\right] .
$$

An analogue of Theorem 3 holds true if, instead of $t$, we take $\tau_{r}$, the first time the process $\left(X_{t}\right)_{t \geq 0}$ exits from $B_{r}$.

Corollary 4. Let $r \in(0,1)$ and $\tau_{r}=\inf \left\{t>0:\left|X_{t}\right|=r\right\}$. If $X_{0}=x$ with $|x|<r$, then

$$
\mathbb{E}^{x}\left[H_{n}^{q, p}\left(\frac{\left\langle X_{\tau_{r}}, x\right\rangle}{\left|X_{\tau_{r}}\right||x|}\right)\right]=H_{n}^{q, p}(1) \cdot \mathbb{E}^{|x|^{2}}\left[\exp \left(-\int_{0}^{\tau_{r}} Q\left(r_{s}\right) d s\right)\right] .
$$

Proof. Let $T_{k}=\inf \left\{t>0: r_{t} \leq 1 / k\right\}$. Because $r_{t}>0$ for all $t>0$ a.s. and this process has continuous trajectories, $T_{k} \rightarrow \infty$ as $k \rightarrow \infty$. To 
simplify notation write $\tau(t, k, r)=t \wedge T_{k} \wedge \tau_{r}$. Because $Z_{t}$ is a martingale, arguing as in the proof of Theorem 3 , for $h \equiv 1$ and the bounded stopping time $\tau(t, k, r)$ we get

$$
\mathbb{E}^{x}\left[H_{n}^{q, p}\left(\frac{\left\langle X_{\tau(t, k, r)}, x\right\rangle}{\left|X_{\tau(t, k, r)}\right||x|}\right)\right]=H_{n}^{q, p}(1) \mathbb{E}^{|x|^{2}}\left[\exp \left(-\int_{0}^{\tau(t, k, r)} Q\left(r_{s}\right) d s\right)\right] .
$$

The function $\exp \left(-\int_{0}^{t} Q\left(r_{s}\right) d s\right)$ is bounded, because $Q$ is positive. If $k \rightarrow \infty$, then $T_{k} \rightarrow \infty$. If we also let $t \rightarrow \infty$, using the dominated convergence theorem, we get the assertion.

9. Poisson kernel of a ball. Now we can compute the coefficients of the expansion of $P_{r}(x, y)$, the Poisson kernel of a ball $B_{r}$. If $X_{0}=x$, then $P_{r}(x, \cdot)$ is the density (with respect to $\sigma_{r}$ ) of the distribution of $X_{\tau_{r}}$. This fact and formula (5) give

$$
\left(\widehat{P}_{r}\right)_{p, q}=\int_{S_{r}} H_{n}^{q, p}\left(\frac{\langle y, x\rangle}{|y||x|}\right) P_{r}(x, y) d \sigma_{r}(y)=\mathbb{E}^{x} H_{n}^{q, p}\left(\frac{\left\langle X_{\tau_{r}}, x\right\rangle}{\left|X_{\tau_{r}}\right||x|}\right) .
$$

The theorem below determines the coefficients $\left(\widehat{P}_{r}\right)_{p, q}$.

THEOREM 5. Let $x \neq 0$ be the starting point of the complex hyperbolic Brownian motion $\left(X_{t}\right)_{t \geq 0}$. For $|x|<r$,

$$
\mathbb{E}^{x}\left[H_{n}^{q, p}\left(\frac{\left\langle X_{\tau_{r}}, x\right\rangle}{\left|X_{\tau_{r}}\right||x|}\right)\right]=H_{n}^{q, p}(1) \cdot\left(\frac{|x|}{r}\right)^{p+q} \cdot \frac{{ }_{2} F_{1}\left(p, q ; p+q+n ;|x|^{2}\right)}{{ }_{2} F_{1}\left(p, q ; p+q+n ; r^{2}\right)} .
$$

Proof. By Corollary 4 we have

$$
\mathbb{E}^{x}\left[H_{n}^{q, p}\left(\frac{\left\langle X_{\tau_{r}}, x\right\rangle}{\left|X_{\tau_{r}}\right||x|}\right)\right]=H_{n}^{q, p}(1) \mathbb{E}^{|x|^{2}}\left[\exp \left(-\int_{0}^{\tau_{r}} Q\left(r_{s}\right) d s\right)\right] .
$$

Observe that the process under the last expectation is real-valued. Let $x$ be a real number, $0<x<1$. The function $\phi(x)=\mathbb{E}^{x}\left[\exp \left(-\int_{0}^{\tau_{r}} Q\left(r_{s}\right) d s\right)\right]$ is the so-called gauge function (see [ChZ, 4.3]) for the Schrödinger operator based on the generator of $r_{t}$ and potential $-Q$, hence, by the general Feynman-Kac theory for the Schrödinger equation [ChZ], $\phi$ is a solution of the appropriate Schrödinger equation (cf. (8) and definition of $Q$ ):

$$
\begin{aligned}
& 4 x(1-x)^{2} \phi^{\prime \prime}(x)+4(1-x)(n-x) \phi^{\prime}(x) \\
& -4(1-x)\left[\frac{\frac{p+q}{2}\left(\frac{p+q}{2}+n-1\right)}{x}-\left(\frac{p-q}{2}\right)^{2}\right] \phi(x)=0 .
\end{aligned}
$$

If we divide both sides of $(35)$ by $4(1-x)$ and next substitute $\phi(x)=$ $x^{(p+q) / 2} y(x)$, we get

$$
x(1-x) y^{\prime \prime}(x)+[p+q+n-(p+q+1) x] y^{\prime}(x)-p q y(x)=0 .
$$


This is the hypergeometric equation (1), hence the general solution of (35) is given in terms of the hypergeometric function ${ }_{2} F_{1}$ and is the following:

$$
\begin{aligned}
y(x)= & x^{(p+q) / 2}\left[c_{1} \cdot{ }_{2} F_{1}(p, q ; p+q+n ; x)\right. \\
& \left.+c_{2} \cdot \frac{2 F_{1}(1-n-q, 1-n-p ; 2-n-p-q ; x)}{x^{p+q+n-1}}\right] .
\end{aligned}
$$

When $x \rightarrow 0$, the expectation $\mathbb{E}^{|x|^{2}}\left[\exp \left(-\int_{0}^{\tau_{r}} Q\left(r_{s}\right) d s\right)\right]$ remains bounded (because $Q$ is positive), so that we are interested in a solution of (36) which is bounded in the neighbourhood of zero. This implies $c_{2}=0$ in the formula (36) and

$$
\phi(x)=c_{1} x^{(p+q) / 2}{ }_{2} F_{1}(p, q ; p+q+n ; x) .
$$

If $X_{t}$ starts from $x$ with $|x|=r$ then $\tau_{r}=0$, hence $\phi\left(r^{2}\right)=1$ and this implies

$$
c_{1}=\frac{1}{r^{p+q} \cdot{ }_{2} F_{1}\left(p, q ; p+q+n ; r^{2}\right)} .
$$

Finally, for $x \in B_{r}$, we get

$$
\phi\left(|x|^{2}\right)=\frac{|x|^{p+q}}{r^{p+q}} \cdot \frac{{ }_{2} F_{1}\left(p, q ; p+q+n ;|x|^{2}\right)}{{ }_{2} F_{1}\left(p, q ; p+q+n ; r^{2}\right)}
$$

and the proof is finished.

REMARK. If $X_{0}=0$ then, by the unitary invariance of the distribution of the complex hyperbolic Brownian motion, the distribution of $X_{\tau_{r}}$ is a unitary invariant probability measure on the sphere $S_{r}$. By [R, 1.4.7], $\sigma_{r} / \omega_{2 n-1}$ is the only measure with this property.

Knowing the coefficients, we can give the expansion of the Poisson kernel.

Theorem 6 (Poisson kernel). Let $0 \neq x \in B_{r}$ and $y \in S_{r}$. Then

$$
P_{r}(x, y)=\frac{1}{r^{2 n-1}} \sum_{p, q=0}^{\infty}\left(\frac{|x|}{r}\right)^{p+q} \frac{{ }_{2} F_{1}\left(p, q ; p+q+n ;|x|^{2}\right)}{{ }_{2} F_{1}\left(p, q ; p+q+n ; r^{2}\right)} H_{n}^{p, q}\left(\frac{\langle y, x\rangle}{|y||x|}\right) .
$$

For every fixed $x \in B_{r}$ the series is convergent absolutely and uniformly for all $y \in S_{r}$.

REMARK. It is possible to get the same expansion by the purely analytical method of Folland $([\mathrm{F}])$. This method is even shorter, but depends heavily on the fact that $P_{r}(x, y)$, as a function of $x$, is $\Delta_{\mathrm{LB}}$-harmonic on the whole $B_{r}$. Our method, exploiting the Itô calculus, seems to be more general and also gives the description of the Green function $G_{r}(x, y)$, which is not continuous on $B_{r}$ (it tends to infinity as $y \rightarrow x$ ). 
Proof of Theorem 6. We will show that the series

$$
\sum_{p, q=0}^{\infty}\left(\frac{|x|}{r}\right)^{p+q} \frac{{ }_{2} F_{1}\left(p, q ; p+q+n ;|x|^{2}\right)}{{ }_{2} F_{1}\left(p, q ; p+q+n ; r^{2}\right)} H_{n}^{p, q}\left(\frac{\langle y, x\rangle}{|y||x|}\right)
$$

is convergent absolutely and uniformly for all $x \in B_{r}$ and $y \in S_{r}$. The proof is a slight modification of that given by Folland [F] for the case $r=1$. We give it here for completeness.

By Proposition 1, for any orthonormal basis $f_{1}, \ldots, f_{D(p, q, n)}$ of $\mathcal{H}^{p, q} \subset$ $L^{2}\left(S_{1}, \sigma_{1}\right)$ and any $x, y \in S_{1}$,

$$
\sum_{j=1}^{D(p, q, n)} f_{j}(x) \overline{f_{j}(y)}=H_{n}^{p, q}(\langle x, y\rangle), \text { so } \sum_{j=1}^{D(p, q, n)}\left|f_{j}(x)\right|^{2}=H_{n}^{p, q}(1)=\frac{D(p, q, n)}{\omega_{2 n-1}} .
$$

Orthogonality of $f_{j}$ 's implies

$$
\begin{aligned}
\int_{S_{1}}\left|H_{n}^{p, q}(\langle x, y\rangle)\right|^{2} d \sigma_{1}(y) & =\sum_{j=1}^{D(p, q, n)} \int_{S_{1}}\left|f_{j}(x)\right|^{2}\left|f_{j}(y)\right|^{2} d \sigma_{1}(y) \\
& =\sum_{j=1}^{D(p, q, n)}\left|f_{j}(x)\right|^{2}=\frac{D(p, q, n)}{\omega_{2 n-1}}=H_{n}^{p, q}(1) .
\end{aligned}
$$

Moreover, changing variables, we get

$$
\begin{aligned}
\int_{S_{r}}\left|H_{n}^{p, q}\left(\frac{\langle y, x\rangle}{|y||x|}\right)\right|^{2} \frac{d \sigma_{r}(y)}{r^{2 n-1}} & =\int_{S_{1}}\left|H_{n}^{p, q}\left(\frac{\langle y, x\rangle}{|y||x|}\right)\right|^{2} d \sigma_{1}(y)=\frac{D(p, q, n)}{\omega_{2 n-1}} \\
& =H_{n}^{p, q}(1) .
\end{aligned}
$$

Because $H_{n}^{p, q}(\langle x, y\rangle)$ is Hermitian symmetric in $x$ and $y$, and for fixed $x$ it is in $\mathcal{H}^{p, q}$ as a function of $y$, by the Schwarz inequality we obtain

$$
\left|H_{n}^{p, q}(\langle y, x\rangle)\right|=\left|\int_{S_{1}} H_{n}^{p, q}(\langle y, z\rangle) H_{n}^{p, q}(\langle z, x\rangle) d \sigma_{1}(z)\right| \leq D(p, q, n) \omega_{2 n-1}^{-1} .
$$

Now,

$$
\begin{aligned}
D(p, q, n) & =\frac{(p+q+n-1)(p+n-2) !(q+n-2) !}{p ! q !(n-1) !(n-2) !} \\
& =\frac{(p+q+n-1) p(p+1) \ldots(p+n-2) q(q+1) \ldots(q+n-2) !}{(n-1) !(n-2) !} \\
& \leq \frac{(p+q+n-1)}{(n-1) !(n-2) !}(p+n-2)^{n-1}(q+n-2)^{n-1} \\
& \leq \frac{1}{(n-1) !(n-2) !}(p+q+n-1)^{2 n-1} .
\end{aligned}
$$


Because $n$ is fixed, we have

$$
1 \leq \frac{p+q+n-1}{p+q+1} \leq n-1 \quad \text { for all } p, q=0,1,2, \ldots,
$$

which implies that

$$
D(p, q, n) \leq \frac{(n-1)^{2 n}}{(n-1) !(n-2) !}(p+q+1)^{2 n}=c_{n}(p+q+1)^{2 n} .
$$

The function ${ }_{2} F_{1}\left(p, q ; p+q+n ;|x|^{2}\right)$ is positive and increasing for $|x| \in$ $(0,1)$ so that

$$
\left(\frac{|x|}{r}\right)^{p+q} \frac{{ }_{2} F_{1}\left(p, q ; p+q+n ;|x|^{2}\right)}{{ }_{2} F_{1}\left(p, q ; p+q+n ; r^{2}\right)} \leq\left(\frac{|x|}{r}\right)^{p+q},
$$

thus

$$
\begin{aligned}
& \sum_{p, q=0}^{\infty}\left(\frac{|x|}{r}\right)^{p+q} \frac{{ }_{2} F_{1}\left(p, q ; p+q+n ;|x|^{2}\right)}{{ }_{2} F_{1}\left(p, q ; p+q+n ; r^{2}\right)}\left|H_{n}^{p, q}\left(\frac{\langle x, y\rangle}{|y||x|}\right)\right| \\
& \leq c_{n} \sum_{p, q=0}^{\infty}\left(\frac{|x|}{r}\right)^{p+q}(p+q+1)^{2 n}=c_{n} \sum_{k=0}^{\infty}\left(\frac{|x|}{r}\right)^{k}(k+1)^{2 n+1}<\infty,
\end{aligned}
$$

because $p+q=k$ for $k+1$ different values of $p$, and $|x|<r$.

The functions $\left(H_{n}^{p, q}(\cdot)\right)_{p, q=0,1, \ldots}$ are orthogonal, hence it is easy to compute the $(p, q)$-coefficient of $P_{r}(x, y)$. We have (recall that $H_{n}^{q, p}(\cdot)=\overline{H_{n}^{p, q}(\cdot)}$ )

$$
\begin{aligned}
\left(\widehat{P}_{r}\right)_{p, q}= & \int_{S_{r}} H_{n}^{q, p}\left(\frac{\langle y, x\rangle}{|y||x|}\right) P_{r}(x, y) d \sigma_{r}(y) \\
= & \int_{S_{r}} H_{n}^{q, p}\left(\frac{\langle y, x\rangle}{|y||x|}\right)\left(\frac{1}{r^{2 n-1}} \sum_{p, q=0}^{\infty}\left(\frac{|x|}{r}\right)^{p+q}\right. \\
& \left.\times \frac{{ }_{2} F_{1}\left(p, q ; p+q+n ;|x|^{2}\right)}{{ }_{2} F_{1}\left(p, q ; p+q+n ; r^{2}\right)} H_{n}^{p, q}\left(\frac{\langle y, x\rangle}{|y||x|}\right)\right) d \sigma_{r}(y) \\
= & \left(\frac{|x|}{r}\right)^{p+q} \frac{{ }_{2} F_{1}\left(p, q ; p+q+n ;|x|^{2}\right)}{{ }_{2} F_{1}\left(p, q ; p+q+n ; r^{2}\right)} \\
& \times \int_{S_{r}} H_{n}^{q, p}\left(\frac{\langle y, x\rangle}{|y||x|}\right) H_{n}^{p, q}\left(\frac{\langle y, x\rangle}{|y||x|}\right) \frac{d \sigma_{r}(y)}{r^{2 n-1}} \\
= & H_{n}^{p, q}(1)\left(\frac{|x|}{r}\right)^{p+q} \frac{{ }_{2} F_{1}\left(p, q ; p+q+n ;|x|^{2}\right)}{{ }_{2} F_{1}\left(p, q ; p+q+n ; r^{2}\right)} .
\end{aligned}
$$

These coefficients are the same as the $(p, q)$-coefficients of the distribution of $X_{\tau_{r}}$, computed in Theorem 5. This implies that the series described in Theorem 6 represents the Poisson kernel of $B_{r}$. 
10. Green function of a ball. In order to compute the Green function of a ball we use the method of Byczkowski and Małecki [BM]. We will compute $G_{r}(x, y)$, the Green function of the ball $B_{r}$, on all spheres $S_{R}$, that is, for fixed $x \in B_{r}$ and all $y \in S_{R}, 0<R<r$. Moreover, because the Green function is symmetric: $G_{r}(x, y)=G_{r}(y, x)$, it is enough to consider the case $|x|<R$.

Below we will use several semigroups and Green operators. Recall that $\left(X_{t}\right)$ denotes the complex hyperbolic Brownian motion and $r_{t}=\left|X_{t}\right|^{2}$. Let $P_{t}^{(r)}$ denote the semigroup of transition probabilities for the process $X_{t}^{B_{r}}$, the hyperbolic Brownian motion killed at the boundary of $B_{r}$. Similarly, let $\left(r_{t}^{(r)}\right)$ be the process $\left(r_{t}\right)$ killed on exiting the interval $\left(0, r^{2}\right)$. Because $X_{t}$ does not hit $0,\left(r_{t}\right)$ exits $\left(0, r^{2}\right)$ through the point $r^{2}$. We denote by $T_{t}^{(r)}$ the Feynman-Kac semigroup based on the process $r_{t}$ and potential $-Q$, with its Feynman-Kac functional $\exp \left(-\int_{0}^{t} Q\left(r_{s}\right) d s\right)$ stopped at the first time the process $\left(r_{t}\right)$ reaches the point $r^{2}$. Let $G_{r}$ denote the Green operator for the process $X_{t}$ and the set $B_{r}$, and let $G_{r}(x, y)$ be the Green function, i.e. the kernel of $G_{r}$. Finally, $V^{(r)}$ will denote the $(-Q)$-Green operator for the semigroup $T_{t}^{(r)}$. For the details of the above definitions the reader may consult [ChZ].

Let $\left(\widehat{G}_{r}\right)_{p, q}(x, R)$ denote the $(p, q)$-coefficient of $G_{r}(x, y)$, defined as in (5), considered as a function of $y \in S_{R}$, with fixed $x \in B_{r}$. Observe that two such coefficients are equal if $\left|x_{1}\right|=\left|x_{2}\right|$. For this reason we will use the notation $\left(\widehat{G}_{r}\right)_{p, q}(|x|, R)$. By formula (5) we have

$$
\left(\widehat{G}_{r}\right)_{p, q}(|x|, R)=\int_{S_{R}} H_{n}^{q, p}\left(\frac{\langle y, x\rangle}{|y||x|}\right) G_{r}(x, y) d \sigma_{R}(y) .
$$

Now we compute these coefficients explicitly. To simplify the formulation of the next theorem, write

$$
\begin{aligned}
F_{p, q}(x) & ={ }_{2} F_{1}(p, q ; p+q+n ; x), \\
G_{p, q}(x) & ={ }_{2} F_{1}(1-n-q, 1-n-p ; 2-n-p-q ; x) .
\end{aligned}
$$

TheOREM 7. Let $G_{r}(x, y)$ be the Green function for the process $\left(X_{t}\right)_{t \geq 0}$ and ball $B_{r}$. Then

$$
\begin{aligned}
& \left(\widehat{G}_{r}\right)_{p, q}(|x|, R) \\
& \quad=\frac{H_{n}^{p, q}(1)|x|^{p+q} F_{p, q}\left(|x|^{2}\right)}{2(p+q+n-1)} R^{p+q}\left(\frac{G_{p, q}\left(R^{2}\right)}{R^{2(p+q+n-1)}}-\frac{G_{p, q}\left(r^{2}\right)}{r^{2(p+q+n-1)}} \cdot \frac{F_{p, q}\left(R^{2}\right)}{F_{p, q}\left(r^{2}\right)}\right) .
\end{aligned}
$$

Proof. In terms of semigroups of operators Theorem 3 reads as follows: for bounded measurable $h: \mathbb{R} \rightarrow \mathbb{R}$,

$$
\left(P_{t}^{(r)} h H_{n}^{q, p}\right)(x)=H_{n}^{q, p}(1)\left(T_{t}^{(r)} h\right)\left(|x|^{2}\right) .
$$


Integrating (39) with respect to time, we get

$$
\int_{0}^{\infty}\left(P_{t}^{(r)} h H_{n}^{q, p}\right)(x) d t=H_{n}^{q, p}(1) \int_{0}^{\infty}\left(T_{t}^{(r)} h\right)\left(|x|^{2}\right) d t,
$$

which, in the language of potential operators, is

$$
\left(G_{r} h H_{n}^{q, p}\right)(x)=H_{n}^{q, p}(1)\left(V^{(r)} h\right)\left(|x|^{2}\right)
$$

Using the fact that $G_{r}(x, y)$ denotes the kernel of the operator $G_{r}$ and integrating in polar coordinates in $\mathbb{C}^{n}$, we can write the left-hand side of (40) as

$$
\begin{aligned}
\left(G_{r} h H_{n}^{q, p}\right)(x) & =\int_{B_{r}} h\left(|y|^{2}\right) H_{n}^{q, p}\left(\frac{\langle y, x\rangle}{|y||x|}\right) G_{r}(x, y) d y \\
& =\int_{0}^{r} h\left(R^{2}\right)\left(\int_{S_{R}} H_{n}^{q, p}\left(\frac{\langle y, x\rangle}{|y||x|}\right) G_{r}(x, y) d \sigma_{R}(y)\right) d R .
\end{aligned}
$$

The quantity $\left(V^{(r)} h\right)\left(|x|^{2}\right)$ on the right-hand side of (40) equals

$$
\begin{aligned}
\left(V^{(r)} h\right)\left(|x|^{2}\right) & =\int_{0}^{r^{2}} h(y) V^{(r)}\left(|x|^{2}, y\right) d y \\
& =\int_{0}^{r} h\left(R^{2}\right) V^{(r)}\left(|x|^{2}, R^{2}\right) 2 R d R
\end{aligned}
$$

where $V^{(r)}\left(|x|^{2}, y\right)$ is the kernel of the operator $V^{(r)}$.

Substituting (42) into the right-hand of (40) and then comparing the right-hand sides of (40) and (41), we get, for measurable bounded functions $h$,

$$
\begin{aligned}
H_{n}^{q, p}(1) \int_{0}^{r} h\left(R^{2}\right) V^{(r)} & \left(|x|^{2}, R^{2}\right) 2 R d R \\
& =\int_{0}^{r} h\left(R^{2}\right)\left(\int_{S_{R}} H_{n}^{q, p}\left(\frac{\langle y, x\rangle}{|y||x|}\right) G_{r}(x, y) d \sigma_{R}(y)\right) d R .
\end{aligned}
$$

This implies

$$
2 H_{n}^{q, p}(1) R V^{(r)}\left(|x|^{2}, R^{2}\right)=\int_{S_{R}} H_{n}^{q, p}\left(\frac{\langle y, x\rangle}{|y||x|}\right) G_{r}(x, y) d \sigma_{R}(y) .
$$

This in turn, together with (38), gives

$$
\left(\widehat{G}_{r}\right)_{p, q}(|x|, R)=2 H_{n}^{q, p}(1) R V^{(r)}\left(|x|^{2}, R^{2}\right) .
$$

The $(-Q)$-Green function $V^{(r)}\left(y, R^{2}\right)$ is $(-Q)$-harmonic in $y$, for $0<y<R$ and for $R<y<r$ (cf. [ChZ]), hence it has to be a solution of (35), the 
Schrödinger equation based on the generator of the process $\left(r_{t}\right)_{t \geq 0}$ and potential $-Q$. We have already found this solution: it is given by the formula (36), hence

$$
\begin{aligned}
& V^{(r)}\left(|x|^{2}, R^{2}\right) \\
& =|x|^{p+q}\left[c_{1}(p, q, R) \cdot{ }_{2} F_{1}\left(p, q ; p+q+n ;|x|^{2}\right)\right. \\
& \left.\quad+\frac{c_{2}(p, q, R)}{|x|^{2(p+q+n-1)}} \cdot{ }_{2} F_{1}\left(1-q-n, 1-p-n ; 2-p-q-n ;|x|^{2}\right)\right] \\
& =|x|^{p+q}\left(c_{1}(p, q, R) F_{p, q}\left(|x|^{2}\right)+\frac{c_{2}(p, q, R)}{|x|^{2(p+q+n-1)}} G_{p, q}\left(|x|^{2}\right)\right) .
\end{aligned}
$$

Because $\lim _{|x|^{2} \rightarrow r^{2}} V^{(r)}\left(|x|^{2}, R^{2}\right)=0$, we have

$$
c_{1}(p, q, R)=-\frac{c_{2}(p, q, R)}{r^{2(p+q+n-1)}} \cdot \frac{G_{p, q}\left(r^{2}\right)}{F_{p, q}\left(r^{2}\right)}
$$

and

$$
\begin{aligned}
& V^{(r)}\left(|x|^{2}, R^{2}\right) \\
& \quad=c_{2}(p, q, R)|x|^{p+q}\left(\frac{G_{p, q}\left(|x|^{2}\right)}{|x|^{2(p+q+n-1)}}-\frac{G_{p, q}\left(r^{2}\right)}{r^{2(p+q+n-1)}} \cdot \frac{F_{p, q}\left(|x|^{2}\right)}{F_{p, q}\left(r^{2}\right)}\right) .
\end{aligned}
$$

By virtue of (43) and (45),

$$
\begin{aligned}
& \left(\widehat{G}_{r}\right)_{p, q}(|x|, R) \\
& \quad=2 H_{n}^{q, p}(1) R \cdot c_{2}(p, q, R)|x|^{p+q}\left(\frac{G_{p, q}\left(|x|^{2}\right)}{|x|^{2(p+q+n-1)}}-\frac{G_{p, q}\left(r^{2}\right)}{r^{2(p+q+n-1)}} \cdot \frac{F_{p, q}\left(|x|^{2}\right)}{F_{p, q}\left(r^{2}\right)}\right) .
\end{aligned}
$$

The Green function is symmetric so that $\left(\widehat{G}_{r}\right)_{p, q}(|x|, R)$ also has this property. Changing the roles of $|x|$ and $R$, we get

$$
\begin{aligned}
& \left(\widehat{G}_{r}\right)_{p, q}(R,|x|) \\
= & 2 H_{n}^{q, p}(1)|x| \cdot c_{2}(p, q,|x|) R^{p+q}\left(\frac{G_{p, q}\left(R^{2}\right)}{R^{2(p+q+n-1)}}-\frac{G_{p, q}\left(r^{2}\right)}{r^{2(p+q+n-1)}} \cdot \frac{F_{p, q}\left(R^{2}\right)}{F_{p, q}\left(r^{2}\right)}\right) .
\end{aligned}
$$

Now we compute the constant $c_{2}(p, q,|x|)$, showing first the following:

LEMMA 8. Let

$$
u(x)=(n+p+q-1) F_{p, q}(x) G_{p, q}(x)+x\left(F_{p, q}^{\prime}(x) G_{p, q}(x)-F_{p, q}(x) G_{p, q}^{\prime}(x)\right) .
$$

Then $u(x)=(n+p+q-1)(1-x)^{n-1}$.

Proof. Recall that, by (1) and the definition, $F_{p, q}$ satisfies the equation

$$
x(1-x) F^{\prime \prime}(x)+(p+q+n-(p+q+1) x) F^{\prime}(x)-p q F(x)=0,
$$


while $G_{p, q}$ satisfies the equation

$$
\begin{aligned}
x(1-x) G^{\prime \prime}(x)+(2-n-p-q-(3-p-q-2 n) x) G^{\prime}(x) \\
-(1-p-n)(1-q-n) G(x)=0 .
\end{aligned}
$$

Let us compute the derivative

$$
\begin{aligned}
u^{\prime}(x)= & (n+p+q-1)\left(F_{p, q}^{\prime}(x) G_{p, q}(x)+F_{p, q}(x) G_{p, q}^{\prime}(x)\right)+F_{p, q}^{\prime}(x) G_{p, q}(x) \\
& -F_{p, q}(x) G_{p, q}^{\prime}(x)+x\left(F_{p, q}^{\prime \prime}(x) G_{p, q}(x)-F_{p, q}(x) G_{p, q}^{\prime \prime}(x)\right) .
\end{aligned}
$$

We can calculate $x(1-x) F_{p, q}^{\prime \prime}(x)$ and $x(1-x) G_{p, q}^{\prime \prime}(x)$ from the above-cited equations. Substituting these quantities, we get

$$
\begin{aligned}
(1-x) u^{\prime}(x)= & (1-n)\left[(n+p+q+1) F_{p, q}(x) G_{p, q}(x)\right. \\
& \left.+x\left(F_{p, q}^{\prime}(x) G_{p, q}(x)-F_{p, q}(x) G_{p, q}^{\prime}(x)\right)\right] \\
= & (1-n) u(x) .
\end{aligned}
$$

Solving the equation $(1-x) u^{\prime}(x)=(1-n) u(x)$, we get $u(x)=C(1-x)^{n-1}$. For $x \rightarrow 0$ the asymptotics of the hypergeometric function and its derivative is well-known [E]; using this asymptotics we get $\lim _{x \rightarrow 0+} u(x)=n+p+q-1$, hence finally $u(x)=(n+p+q-1)(1-x)^{n-1}$.

Using Lemma 8 we can compute the derivative of the function

$$
R^{p+q}\left(\frac{G_{p, q}\left(R^{2}\right)}{R^{2(p+q+n-1)}}-\frac{G_{p, q}\left(r^{2}\right)}{r^{2(p+q+n-1)}} \cdot \frac{F_{p, q}\left(R^{2}\right)}{F_{p, q}\left(r^{2}\right)}\right)
$$

with respect to $R$ :

$$
\begin{aligned}
\left(-\left.\frac{d}{d R}\right|_{R=r}\right) R^{p+q}\left(\frac{G_{p, q}\left(R^{2}\right)}{R^{2(p+q+n-1)}}\right. & \left.-\frac{G_{p, q}\left(r^{2}\right)}{r^{2(p+q+n-1)}} \cdot \frac{F_{p, q}\left(R^{2}\right)}{F_{p, q}\left(r^{2}\right)}\right) \\
& =\frac{2(p+q+n-1)\left(1-r^{2}\right)^{n-1}}{r^{p+q+2 n-1} F_{p, q}\left(r^{2}\right)} .
\end{aligned}
$$

On the other hand, by the Green formula (see Lemma 11 in the Appendix), for $y \in S_{1}$ we have

$$
\left(-\left.\frac{d}{d R}\right|_{R=r}\right) G_{r}(x, R y)=\left(1-r^{2}\right)^{n-1} P_{r}(x, r y) .
$$

From this fact and (38), for $|y|=1$ and $|x|<r$ we obtain

$$
\begin{aligned}
\left(-\left.\frac{d}{d R}\right|_{R=r}\right)\left(\widehat{G}_{r}\right)_{p, q}(|x|, R) & \\
= & \left(-\left.\frac{d}{d R}\right|_{R=r}\right) \int_{S_{1}} H_{n}^{q, p}\left(\frac{\langle y, x\rangle}{|x||y|}\right) G_{r}(x, R y) d \sigma_{1}(y) \\
& =\int_{S_{1}}\left(-\left.\frac{d}{d R}\right|_{R=r}\right) H_{n}^{q, p}\left(\frac{\langle y, x\rangle}{|x||y|}\right) G_{r}(x, R y) d \sigma_{1}(y)
\end{aligned}
$$




$$
\begin{aligned}
& =\left(1-r^{2}\right)^{n-1} \int_{S_{1}} H_{n}^{q, p}\left(\frac{\langle y, x\rangle}{|x||y|}\right) P_{r}(x, r y) d \sigma_{1}(y) \\
& =\frac{\left(1-r^{2}\right)^{n-1}}{r^{2 n-1}} \int_{S_{r}} H_{n}^{q, p}\left(\frac{\langle y, x\rangle}{|x||y|}\right) P_{r}(x, y) d \sigma_{r}(y) \\
& =\frac{\left(1-r^{2}\right)^{n-1}}{r^{2 n-1}} H_{n}^{q, p}(1) \cdot \frac{|x|^{p+q}}{r^{p+q}} \cdot \frac{F_{p, q}\left(|x|^{2}\right)}{F_{p, q}\left(r^{2}\right)}
\end{aligned}
$$

where in the last equality we have used Theorem 6 .

Now we can compare the derivatives: formula (48) gives

$$
\left(-\left.\frac{d}{d R}\right|_{R=r}\right)\left(\widehat{G}_{r}\right)_{p, q}(|x|, R)=\frac{\left(1-r^{2}\right)^{n-1}}{r^{2 n-1}} H_{n}^{q, p}(1) \frac{|x|^{p+q}}{r^{p+q}} \cdot \frac{F_{p, q}\left(|x|^{2}\right)}{F_{p, q}\left(r^{2}\right)},
$$

while (46) and (47) imply

$$
\begin{aligned}
\left(-\left.\frac{d}{d R}\right|_{R=r}\right) & \left(\widehat{G}_{r}\right)_{p, q}(|x|, R) \\
& =2 H_{n}^{q, p}(1)|x| c_{2}(p, q,|x|) \cdot \frac{2(p+q+n-1)\left(1-r^{2}\right)^{n-1}}{r^{p+q+2 n-1} F_{p, q}\left(r^{2}\right)} .
\end{aligned}
$$

Comparing the right-hand sides of the last two expressions, we get

$$
\begin{aligned}
& \frac{\left(1-r^{2}\right)^{n-1} H_{n}^{q, p}(1)|x|^{p+q} F_{p, q}\left(|x|^{2}\right)}{r^{2 n-1} r^{p+q} F_{p, q}\left(r^{2}\right)} \\
& =\frac{4(p+q+n-1) H_{n}^{q, p}(1)|x| c_{2}(p, q,|x|)\left(1-r^{2}\right)^{n-1}}{r^{p+q+2 n-1} F_{p, q}\left(r^{2}\right)},
\end{aligned}
$$

hence

$$
|x| c_{2}(p, q,|x|)=\frac{|x|^{p+q} F_{p, q}\left(|x|^{2}\right)}{4(p+q+n-1)},
$$

which, by (46), finally gives

$$
\begin{aligned}
& \left(\widehat{G}_{r}\right)_{p, q}(R,|x|) \\
& \quad=\frac{H_{n}^{q, p}(1)|x|^{p+q} F_{p, q}\left(|x|^{2}\right)}{2(p+q+n-1)} R^{p+q}\left(\frac{G_{p, q}\left(R^{2}\right)}{R^{2(p+q+n-1)}}-\frac{G_{p, q}\left(r^{2}\right)}{r^{2(p+q+n-1)}} \cdot \frac{F_{p, q}\left(R^{2}\right)}{F_{p, q}\left(r^{2}\right)}\right) .
\end{aligned}
$$

Having the coefficients, we can write down the expansion of the Green function.

Theorem 9 (Green function). Let $x, y \in B_{r}$. If $x \neq 0$ and $|x|<|y|$, then

$$
\begin{aligned}
G_{r}(x, y)= & \frac{1}{2 r^{2 n-1}} \sum_{p, q=0}^{\infty} \frac{|x|^{p+q}|y|^{p+q}}{p+q+n-1} F_{p, q}\left(|x|^{2}\right)\left(\frac{G_{p, q}\left(|y|^{2}\right)}{|y|^{2(p+q+n-1)}}\right. \\
& \left.-\frac{G_{p, q}\left(r^{2}\right)}{r^{2(p+q+n-1)}} \cdot \frac{F_{p, q}\left(|y|^{2}\right)}{F_{p, q}\left(r^{2}\right)}\right) H_{n}^{p, q}\left(\frac{\langle x, y\rangle}{|x||y|}\right) .
\end{aligned}
$$


For fixed $x$ the series is uniformly convergent on every sphere $S_{R}$ with $|x|<$ $R<r$.

Proof. To show the uniform convergence, we can estimate the series as follows:

$$
\begin{aligned}
& \mid \sum_{p, q=0}^{\infty} \frac{|x|^{p+q}|y|^{p+q}}{p+q+n-1} F_{p, q}\left(|x|^{2}\right) \\
& \times\left(\frac{G_{p, q}\left(|y|^{2}\right)}{|y|^{2(p+q+n-1)}}-\frac{G_{p, q}\left(r^{2}\right)}{r^{2(p+q+n-1)}} \cdot \frac{F_{p, q}\left(|y|^{2}\right)}{F_{p, q}\left(r^{2}\right)}\right) H_{n}^{p, q}\left(\frac{\langle x, y\rangle}{|x||y|}\right) \mid \\
& \leq \frac{c}{|y|^{2 n-2}} \sum_{p, q=0}^{\infty} \frac{|x|^{p+q}}{|y|^{p+q}} H_{n}^{p, q}(1)\left|F_{p, q}\left(|x|^{2}\right) G_{p, q}\left(|y|^{2}\right)\right| \\
& +\frac{c}{r^{2 n-2}} \sum_{p, q=0}^{\infty} \frac{|x|^{p+q}|y|^{p+q}}{r^{2(p+q)}} H_{n}^{p, q}(1)\left|F_{p, q}\left(|x|^{2}\right) G_{p, q}\left(r^{2}\right)\right|,
\end{aligned}
$$

because $F_{p, q}$ is positive and increasing on $(0,1)$, which implies that $F_{p, q}\left(|x|^{2}\right) / F_{p, q}\left(r^{2}\right) \leq 1$.

Below in Lemma 10 we will show that, except for the case $p q=0, G_{p, q}^{\prime}<0$ on $(0,1)$ and $G_{p, q}(1)=0$. These facts immediately imply that $G_{p, q}>0$ on $(0,1)$ and $x\left(F_{p, q}^{\prime}(x) G_{p, q}(x)-F_{p, q}(x) G_{p, q}^{\prime}(x)\right)>0$, hence by Lemma 8 we have

$$
0<F_{p, q}(x) G_{p, q}(x)<(1-x)^{n-1} \leq 1 \quad \text { for } x \in(0,1) .
$$

This, together with the fact that $F_{p, q}$ is positive and increasing, yields

$$
\begin{gathered}
\left|F_{p, q}\left(|x|^{2}\right) G_{p, q}\left(|y|^{2}\right)\right|<F_{p, q}\left(|y|^{2}\right) G_{p, q}\left(|y|^{2}\right) \leq 1, \\
\left|F_{p, q}\left(|x|^{2}\right) G_{p, q}\left(r^{2}\right)\right|<F_{p, q}\left(r^{2}\right) G_{p, q}\left(r^{2}\right) \leq 1 .
\end{gathered}
$$

By the definition, $G_{0, q}$ is bounded on every interval $[\varepsilon, 1]$ and $F_{0, q} \equiv 1$, hence $F_{0, q} G_{0, q}$ is also bounded on $[\varepsilon, 1]$.

Recall that $0<|x|<|y|<r$ by our assumption and put $\varrho=|x| /|y|$ in the case of the first series and $\varrho=|x||y| / r^{2}$ for the second. In the proof of Theorem 5 we showed that for any $0<\varrho<1$ we have $\sum \varrho^{p+q} H_{n}^{p, q}(1)<\infty$, hence both series are absolutely and uniformly convergent.

The family $\left(H_{n}^{p, q}(\cdot)\right)_{p, q=0}^{\infty}$ is orthogonal, hence we can compute (just as we did for the Poisson kernel in the proof of Theorem 6) the $(p, q)$-coefficients of the function defined by the series. Because the coefficients are equal to those computed in Theorem 7 for the Green function, the series given in Theorem 9 represents $G_{r}(x, y)$, the Green function of $B_{r}$.

Now we will prove the above-mentioned properties of $G_{p, q}$. 
LEMMA 10. For non-negative integers $p, q$ and $n \geq 2$ let

$$
G_{p, q}(x)={ }_{2} F_{1}(1-n-q, 1-n-p ; 2-n-p-q ; x) .
$$

If $p q>0$ then $G_{p, q}^{\prime}(x)<0$ for $x \in(0,1)$ and $G_{p, q}(1)=0$.

Proof. We can assume that $q \leq p$. The Kummer relation mentioned in the Preliminaries implies that

${ }_{2} F_{1}(1-n-q, 1-n-p ; 2-n-p-q ; x)=(1-x)^{n} \cdot{ }_{2} F_{1}(1-p, 1-q ; 2-n-p-q ; x)$.

Since $f(x)=(1-x)^{n}$ is decreasing on $(0,1), f(1)=0$ and ${ }_{2} F_{1}(1-p, 1-q$; $2-n-p-q ; x)$ is a polynomial, it follows that $G_{p, q}(1)=0$ and it is enough to show that $h_{p, q}(x)={ }_{2} F_{1}(1-p, 1-q ; 2-n-p-q ; x)$ has negative derivative for $0<x<1$.

The function $h_{p, q}(x)$ is a polynomial in $x$ of degree $q-1$ (recall that we assumed $0<q \leq p$ ) and for $k=1, \ldots, q-1$ its $k$ th derivative is

$$
\frac{d^{k}}{d x^{k}} h_{p, q}(x)=\frac{(1-p)_{k}(1-q)_{k}}{(2-n-p-q)_{k}} \cdot{ }_{2} F_{1}(1-p+k, 1-q+k ; 2-n-p-q+k ; x) \text {. }
$$

In [V, computations of $C_{2}$ on p. 132], the following formula was proved:

$$
\begin{aligned}
{ }_{2} F_{1}(1-p+k, 1-q+k ; 2-n- & -q+k ; 1) \\
& =\frac{(n+p-1) !(n+q-1) !}{(n+k) !(n+p+q-2+k) !} .
\end{aligned}
$$

Now observe that for fixed $n, q$ and $p$ the sequence $\left(\frac{(1-p)_{k}(1-q)_{k}}{(2-n-p-q)_{k}}\right)_{k=1}^{q-1}$ has alternating signs. Indeed, let $q>0$ be even. Then

$$
\frac{d^{q-1}}{d x^{q-1}} h_{p, q}(1)=\frac{(1-p)_{q-1}(1-q)_{q-1}}{(2-n-p-q)_{q-1}} \cdot{ }_{2} F_{1}(q-p, 0 ; 1-n-p ; 1)<0,
$$

because there are an odd number of terms in the denominator, they are all negative and ${ }_{2} F_{1}(q-p, 0 ; 1-n-p ; 1)=1$. This implies that $d^{q-2} h_{p, q} / d x^{q-2}$ is decreasing and since its value at $x=1$ is positive by (49), this derivative is positive. This implies that the derivative of order $q-3$ is decreasing, and as its value at $x=1$ is positive, $d^{q-3} h_{p, q} / d x^{q-3}$ is positive. By induction we infer that $d h_{p, q} / d x$ is negative, so that $h_{p, q}$ is decreasing, as required.

If $q$ is odd then $\frac{d^{q-1}}{d x^{q-1}} h_{p, q}(1)>0$ and the analogous reasoning gives the same result.

REMARK. The formula in Theorem 9 is very similar to the one obtained by Byczkowski and Małecki $[\mathrm{BM}]$ for the case of the real hyperbolic space $\mathbb{D}_{n}$. Instead of our $F_{p, q}$ their representation uses the hypergeometric function ${ }_{2} F_{1}(p,(2-n) / 2 ; p+n / 2 ; x)$ and for even $n$ this function is a polynomial. Using this fact they managed to find sums of the analogous series describing $P_{r}(x, y)$ and $G_{r}(x, y)$ for $n=4$ and $n=6$. It seems hard to get such compact 
formulas in the complex hyperbolic case, because for all $n \geq 2$ the functions $F_{p, q}$ are given by infinite series.

Appendix: gradient of a function and directional derivative in the complex hyperbolic space. Consider the unit ball $B_{1}=\left\{z \in \mathbb{C}^{n}\right.$ : $|z|<1\}$ with the Bergman (Hermitian) metric, given by the matrix with entries

$$
h_{i j}=\frac{\left(1-|z|^{2}\right) \delta_{i j}+\bar{z}_{i} z_{j}}{\left(1-|z|^{2}\right)^{2}}, \quad i, j=1, \ldots, n
$$

(cf. $[\mathrm{K}]$ or $[\mathrm{S}]$, where the second exponent in $\left(1-|z|^{2}\right)^{2}$ is missing).

The inverse matrix $\left[h^{i j}\right]$ is given by the formula [S]

$$
h^{i j}=\left(1-|z|^{2}\right)\left(\delta_{i j}-\bar{z}_{i} z_{j}\right), \quad i, j=1, \ldots, n .
$$

Because we want to use the Green formula, involving derivatives in the normal direction, we describe this metric in the language of the Riemannian metric in the unit ball of $\mathbb{R}^{2 n}$. By the well-known identification between the structures of $\mathbb{C}^{n}$ and $\mathbb{R}^{2 n}([\mathrm{KN}],[\mathrm{R}])$ we have the following:

1) If $z=\left(z_{1}, \ldots, z_{n}\right) \in \mathbb{C}^{n}$ and $z_{k}=x_{k}+i y_{k}$ then

$$
\mathbb{C}^{n} \ni z \leftrightarrow\left(x_{1}, \ldots, x_{n}, y_{1}, \ldots, y_{n}\right) \in \mathbb{R}^{2 n} .
$$

2) Let $M_{\mathbb{C}^{n}}$ be the matrix of a linear transformation of $\mathbb{C}^{n}$. If $M_{\mathbb{C}^{n}}=$ $\Re M_{\mathbb{C}^{n}}+i \Im M_{\mathbb{C}^{n}}=A+i B$ then

$$
M_{\mathbb{R}^{2 n}}=\left[\begin{array}{cc}
A & B \\
-B & A
\end{array}\right]
$$

is the matrix of the corresponding transformation of $\mathbb{R}^{2 n}$.

Length of a tangent vector. Now we want to look at the unit ball in $\mathbb{C}^{n}$ with its Hermitian metric as a subset of $\mathbb{R}^{2 n}$ with the Riemannian metric. By the above, the Riemannian metric $g_{i j}, i, j=1, \ldots, 2 n$, in the unit ball of $\mathbb{R}^{2 n}$ is given by the matrix $\left[\begin{array}{cc}A & B \\ -B & A\end{array}\right]$, where

$$
\begin{array}{ll}
a_{i j}=\frac{\left(1-|z|^{2}\right) \delta_{i j}+\Re\left(\bar{z}_{i} z_{j}\right)}{\left(1-|z|^{2}\right)^{2}}, & i, j=1, \ldots, n, \\
b_{i j}=\frac{\Im\left(\bar{z}_{i} z_{j}\right)}{\left(1-|z|^{2}\right)^{2}}, & i, j=1, \ldots, n .
\end{array}
$$

Let $T_{\left(x_{1}, \ldots, x_{n}, y_{1}, \ldots, y_{n}\right)}$ denote the tangent space at the point $\left(x_{1}, \ldots, x_{n}\right.$, $\left.y_{1}, \ldots, y_{n}\right)$ and let $v \in T_{\left(x_{1}, \ldots, x_{n}, y_{1}, \ldots, y_{n}\right)}$. Then the form determining the length of $v=\left(v_{1}, \ldots, v_{2 n}\right)$ is given by the formula

$$
d s^{2}=\left[\begin{array}{lll}
v_{1} & \ldots & v_{2 n}
\end{array}\right]\left[\begin{array}{cc}
A & B \\
-B & A
\end{array}\right]\left[\begin{array}{lll}
v_{1} & \ldots & v_{2 n}
\end{array}\right]^{T} .
$$


In particular we get (after a long computation)

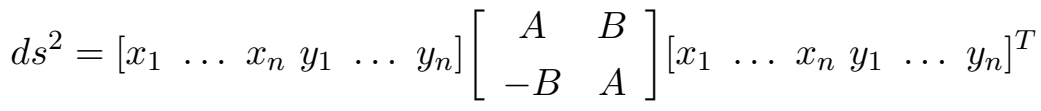

$$
\begin{aligned}
& =\frac{x_{1}^{2}+\cdots+x_{n}^{2}+y_{1}^{2}+\cdots+y_{n}^{2}}{\left(1-x_{1}^{2}-\cdots-x_{n}^{2}-y_{1}^{2}-\cdots-y_{n}^{2}\right)^{2}} \text {, }
\end{aligned}
$$

so that

$$
\left|\left(x_{1}, \ldots, x_{n}, y_{1}, \ldots, y_{n}\right)\right|_{R}=\frac{\left|\left(x_{1}, \ldots, x_{n}, y_{1}, \ldots, y_{n}\right)\right|_{E}}{1-\left|\left(x_{1}, \ldots, x_{n}, y_{1}, \ldots, y_{n}\right)\right|_{E}^{2}},
$$

where $|v|_{E}$ denotes the Euclidean and $|v|_{R}$ the Riemannian length of a vector $v$.

The same result can be obtained without computations: the Bergman metric is invariant with respect to unitary transformations of the unit ball and the group $U(n)$ acts transitively on the spheres $S_{r}, 0<r<1$. Let $z \in \mathbb{C}^{n}$ with $|z|_{E}=r<1$ and choose $A_{z} \in U(n)$ such that $A_{z}(z)=(r, 0, \ldots, 0)$ $\in \mathbb{C}^{n}$. This implies that the length of any vector $v$ in $T_{z}=T_{\left(x_{1}, \ldots, x_{n}, y_{1}, \ldots, y_{n}\right)}$ is the same as the length of $A_{z}(v)$ in $T_{(r, 0, \ldots, 0)}$. In particular the length of $z=\left(x_{1}, \ldots, x_{n}, y_{1}, \ldots, y_{n}\right)$ is the same as the length of $(r, 0, \ldots, 0)$ in $T_{(r, 0, \ldots, 0)}$. But we can identify $(r, 0, \ldots, 0) \in \mathbb{C}^{n}$ with $r \in \mathbb{C}^{1}$. The form of the Bergman metric $h_{i j}$ implies that both vectors have the same length and in the case $n=1$ the Bergman metric in the unit disc of $\mathbb{C}$ is the same as the Poincaré metric, so that

$$
|(r, 0, \ldots, 0)|_{R}=\frac{|(r, 0, \ldots, 0)|_{E}}{1-r^{2}}
$$

Derivative in the normal direction. Let $0<r<1$ and fix a point $\left(x_{1}, \ldots, x_{n}, y_{1}, \ldots, y_{n}\right)$ with Euclidean norm $r$, i.e. a point from the sphere $S_{r}$. For a differentiable function $f$ we want to compute the directional derivative $\partial f / \partial \mathbf{n}$, where $\mathbf{n}$ is the normal vector at $\left(x_{1}, \ldots, x_{n}, y_{1}, \ldots, y_{n}\right)$ and has Euclidean length 1 . In order to use the formula

$$
\frac{\partial f}{\partial \mathbf{n}}=\langle\operatorname{grad} f, \mathbf{n}\rangle
$$

we have to know grad $f$ and the length of $\mathbf{n}$ (which we have already computed). Now we compute the gradient of $f$. Let $\left[g^{i j}\right]$ be the matrix inverse to $\left[g_{i j}\right]$ and let $\left(U,\left(x_{1}, \ldots, x_{2 n}\right)\right)$ be a local coordinate system. Then by the well-known formula for Riemannian manifolds ([KN], [Ch])

$$
\operatorname{grad} f=\sum_{i, j=1}^{2 n}\left(g^{i j} \frac{\partial f}{\partial x_{j}}\right) \frac{\partial}{\partial x_{i}}
$$

where $\left(\partial / \partial x_{i}\right)_{i=1, \ldots, 2 n}$ is a basic vector field. In the case we consider, the 
inverse matrix $\left[g^{i j}\right]$ at $\left(x_{1}, \ldots, x_{n}, y_{1}, \ldots, y_{n}\right)$ is

$$
\left[g^{i j}\right]_{i, j=1, \ldots, 2 n}=\left[\begin{array}{cc}
C & D \\
-D & C
\end{array}\right],
$$

where

$$
\begin{array}{ll}
c^{i j}=\left(1-|z|^{2}\right)\left(\delta_{i j}-\Re\left(\bar{z}_{i} z_{j}\right)\right), & i, j=1, \ldots, n, \\
d^{i j}=\left(1-|z|^{2}\right)\left(-\Im\left(\bar{z}_{i} z_{j}\right)\right), & i, j=1, \ldots, n .
\end{array}
$$

Putting $z_{k}=x_{k}+i y_{k}, k=1, \ldots, n$, with $|z|^{2}=\sum_{i=1}^{n}\left(x_{i}^{2}+y_{i}^{2}\right)=r^{2}$, we get, for $i, j=1, \ldots, n$,

$$
\begin{aligned}
c^{i j} & =\left(1-\sum_{k=1}^{n} x_{k}^{2}-\sum_{k=1}^{n} y_{k}^{2}\right)\left(\delta_{i j}-x_{i} x_{j}-y_{i} y_{j}\right)=\left(1-r^{2}\right)\left(\delta_{i j}-x_{i} x_{j}-y_{i} y_{j}\right), \\
d^{i j} & =\left(1-\sum_{k=1}^{n} x_{k}^{2}-\sum_{k=1}^{n} y_{k}^{2}\right)\left(-x_{i} y_{j}+x_{j} y_{i}\right)=\left(1-r^{2}\right)\left(-x_{i} y_{j}+x_{j} y_{i}\right) .
\end{aligned}
$$

Let $\mathbf{n}$ be the normal vector at $\left(x_{1}, \ldots, x_{n}, y_{1}, \ldots, y_{n}\right) \in S_{r}$. Then $\mathbf{n}=$ $\left(x_{1}, \ldots, x_{n}, y_{1}, \ldots, y_{n}\right)$. By the previous computation its length is equal to $|\mathbf{n}|_{R}=|\mathbf{n}|_{E} /\left(1-r^{2}\right)$. Thus

$$
\begin{aligned}
\langle\operatorname{grad} & f, \mathbf{n}\rangle=\frac{1}{1-r^{2}}\left\langle\operatorname{grad} f,\left(x_{1}, \ldots, x_{n}, y_{1}, \ldots, y_{n}\right)\right\rangle \\
= & \frac{1}{1-r^{2}}\left\langle\left(\sum_{j=1}^{n} g^{i j} \frac{\partial f}{\partial x_{j}}+\sum_{j=1}^{n} g^{i n+j} \frac{\partial f}{\partial y_{j}}\right)_{i=1}^{2 n},\left(x_{1}, \ldots, x_{n}, y_{1}, \ldots, y_{n}\right)\right\rangle \\
= & \sum_{i=1}^{n} C_{i} \frac{\partial f}{\partial x_{i}}+\sum_{i=1}^{n} D_{i} \frac{\partial f}{\partial y_{i}}
\end{aligned}
$$

Now we compute the coefficients $C_{i}$ and $D_{i}$, using the form of the matrix $\left[g^{i j}\right]=\left[\begin{array}{cc}C & D \\ -D & C\end{array}\right]$. For $i=1, \ldots, n$ we have

$$
\begin{aligned}
C_{i} & =\sum_{j=1}^{n}\left(\delta_{j i}-x_{j} x_{i}-y_{j} y_{i}\right) x_{j}+\sum_{j=1}^{n}\left(x_{j} y_{i}-x_{i} y_{j}\right) y_{j} \\
& =x_{i}\left(1-\sum_{j=1}^{n} x_{j}^{2}-\sum_{j=1}^{n} y_{j}^{2}\right)=\left(1-r^{2}\right) x_{i}, \\
D_{i} & =\sum_{j=1}^{n}\left(-x_{j} y_{i}+x_{i} y_{j}\right) x_{j}+\sum_{j=1}^{n}\left(\delta_{j i}-x_{j} x_{i}-y_{j} y_{i}\right) y_{j} \\
& =y_{i}\left(1-\sum_{j=1}^{n} x_{j}^{2}-\sum_{j=1}^{n} y_{j}^{2}\right)=\left(1-r^{2}\right) y_{i} .
\end{aligned}
$$


Finally, the above formulae give

$$
\langle\operatorname{grad} f, \mathbf{n}\rangle=\left(1-r^{2}\right)\left\langle\nabla f,\left(x_{1}, \ldots, x_{n}, y_{1}, \ldots, y_{n}\right)\right\rangle \text {. }
$$

In the space $\mathbb{R}^{2 n}$ we have ( $\lambda$ denotes here the Lebesgue measure):

$$
\lambda(B(0, r))=\int_{0}^{r} \omega_{2 n-1} \varrho^{2 n-1} d \varrho=\frac{\omega_{2 n-1}}{2 n} r^{2 n},
$$

whence the measure of the sphere $S_{r}$, which is the derivative of $\lambda(B(0, r))$, is $\omega_{2 n-1} r^{2 n-1}$.

In the unit ball of $\mathbb{R}^{2 n}$ equipped with the Riemann metric (inherited from the Bergman metric in the unit ball of $\mathbb{C}^{n}$ ) we have the following Riemannian volume $V$ of the ball (cf. [R, Thm. 2.2.6(ii)]):

$$
V(B(0, r))=\int_{0}^{r} \omega_{2 n-1} \frac{\varrho^{2 n-1}}{\left(1-\varrho^{2}\right)^{n+1}} d \varrho .
$$

This implies that the Riemannian measure of the sphere $S_{r}$ is equal to

$$
A(r)=\lim _{\varepsilon \rightarrow 0+} \frac{V(B(0, r+\varepsilon))-V(B(0, r))}{\varepsilon /\left(1-r^{2}\right)}=\omega_{2 n-1} \frac{r^{2 n-1}}{\left(1-r^{2}\right)^{n}},
$$

because the interval (in the unit ball of $\mathbb{C}^{n}$ ) with endpoints $(r, 0, \ldots, 0)$ and $(r+\varepsilon, 0, \ldots, 0)$ has length $\varepsilon /\left(1-r^{2}\right)+o(\varepsilon)$. Thus the measure on $S_{r}$, induced by the Riemannian measure, is equal to the Euclidean measure divided by $\left(1-r^{2}\right)^{n}$.

LEMMA 11. Consider $B_{1}$, the unit ball in $\mathbb{C}^{n}$, equipped with the Bergman metric and let $\Delta_{\mathrm{LB}}$ be the Laplace-Beltrami operator on this Riemannian manifold. Fix $r \in(0,1)$ and denote by $P_{r}(x, y)$ the Poisson kernel, and by $G_{r}(x, y)$ the Green function of $B_{r}$ with respect to $\Delta_{\mathrm{LB}}$. Then, in the above notation,

$$
-\left.\frac{d}{d R}\right|_{R=r}\left(G_{r}(x, R y)\right)_{E}=\left(1-r^{2}\right)^{n-1} P_{r}(x, r y),
$$

where the subscript $E$ in $\left(G_{r}(x, R y)\right)_{E}$ denotes that this quantity is computed with respect to the Euclidean metric.

Proof. By Theorem 8 of [Ch, p. 174], for $u$ harmonic in $B_{r}$ and $u \in$ $C^{1}\left(\bar{B}_{r}\right)$

$$
-u(x)=\int_{\partial B_{r}}\left(\frac{\partial G_{r}}{\partial v_{w}}\right)_{R}(x, w) u(w) d A(w),
$$

where now the subscript $R$ denotes that the derivative is computed with respect to the Riemannian metric. We want to use the Euclidean metric and then, by (50), for $w \in S_{r}$ we have

$$
d A(w)=\frac{d \sigma_{r}(w)}{\left(1-r^{2}\right)^{n}}
$$


and the Riemannian directional derivative is $1-r^{2}$ times the Euclidean one, hence

$$
-u(x)=\frac{1}{\left(1-r^{2}\right)^{n-1}} \int_{\partial B_{r}}\left(\frac{\partial G}{\partial v_{w}}\right)_{E}(x, w) u(w) d \sigma_{r}(w) .
$$

Because the function $\partial G / \partial v_{w}$ reproduces harmonic functions, it has to be the Poisson kernel. This gives

$$
-\left.\frac{d}{d R}\right|_{R=r}\left(G_{D}(x, R y)\right)_{E}=\left(1-r^{2}\right)^{n-1} P_{r}(x, r y) .
$$

Acknowledgments. The results of this paper were presented during the conference "Stochastic Processes with Jumps", May 2-9, 2006, Angers, France. The author is grateful to the organizers, especially Professor Piotr Graczyk, for this oportunity.

The author also expresses his gratitude to the referee, whose hints how to shorten the proof of the independence of the processes $W^{(1)}, W^{(2)}$ and $W^{(3)}$ improved the paper.

\section{References}

[BM] T. Byczkowski and J. Małecki, Poisson kernel and Green function of the ball in real hyperbolic spaces, Potential Anal. 27 (2007), 1-26.

[Ch] I. Chavel, Eigenvalues in Riemannian Geometry, Academic Press, London, 1984.

[ChZ] K. L. Chung and Z. X. Zhao, From Brownian Motion to Schrödinger's Equation, Grundlehren Math. Wiss. 312, Springer, Berlin, 1995.

[E] A. Erdélyi et al., Higher Transcendental Functions, Vol. I, McGraw-Hill, 1953.

[F] G. B. Folland, Spherical harmonic expansion of the Poisson-Szegö kernel for the ball, Proc. Amer. Math. Soc. 47 (1975), 401-408.

[IMcK] K. Itô and H. P. McKean, Diffusion Processes and their Sample Paths, Grundlehren Math. Wiss. 125, Springer, Berlin, 1965.

[KN] S. Kobayashi and K. Nomizu, Foundations of Differential Geometry, Vol. II, Interscience Publ., 1969.

[K] T. H. Koornwinder, The addition formula for Jacobi polynomials II, Math. Centrum Amsterdam Afd. Toegep. Wisk. Report TW 133, 1972; http://staff.science. uva.nl/ $\sim$ thk/art/1972/addition2.pdf.

[Kr] S. G. Krantz, Function Theory of Several Complex Variables, Wiley, New York, 1982.

[M] H. Matsumoto, Closed form formulae for the heat kernels and the Green functions for the Laplacians on the symmetric spaces of rank one, Bull. Sci. Math. 125 (2001), 553-581.

[PR] E. J. Pauwels and L. C. G. Rogers, Skew-product decompositions of Brownian motion, in: Contemp. Math. 73, Amer. Math. Soc., 1988, 237-262.

[RW] L. C. G. Rogers and D. Williams, Diffusions, Markov Processes and Martingales. Volume 2: Itô Calculus, Wiley, Chichester, 1987.

[R] W. Rudin, Function Theory in the Unit Ball of $\mathbb{C}^{n}$, Springer, 1980.

[S] E. M. Stein, Boundary Behavior of Holomorphic Functions of Several Complex Variables, Math. Notes 11, Princeton Univ. Press, 1972. 
[V] R. Vidūnas, Degenerate Gauss hypergeometric function, Kyushu J. Math. 61 (2007), 109-135.

[W] D. Williams, Diffusions, Markov Processes and Martingales. Volume 1: Foundations, Wiley, Chichester, 1979.

Institute of Mathematics and Computer Science

Wrocław University of Technology

Wybrzeże Wyspiańskiego 27

50-370 Wrocław, Poland

E-mail: Tomasz.Zak@pwr.wroc.pl

Received January 6, 2007

Revised version July 23, 2007 